\title{
CONSTANT TERM IDENTITIES AND POINCARÉ POLYNOMIALS
}

\author{
GYULA KÁROLYI, ALAIN LASCOUX, AND S. OLE WARNAAR
}

\begin{abstract}
In 1982 Macdonald published his now famous constant term conjectures for classical root systems. This paper begins with the almost trivial observation that Macdonald's constant term identities admit an extra set of free parameters, thereby linking them to Poincaré polynomials. We then exploit these extra degrees of freedom in the case of type A to give the first proof of Kadell's orthogonality conjecture - a symmetric function generalisation of the $q$-Dyson conjecture or Zeilberger-Bressoud theorem.

Key ingredients in our proof of Kadell's orthogonality conjecture are multivariable Lagrange interpolation, the scalar product for Demazure characters and $(0,1)$-matrices.
\end{abstract}

\section{INTRODUCTION AND SUMMARY OF RESULTS}

Given a finite real reflection group $W$, the classical Poincaré polynomial $W(t)$ is defined as [5, 18,

$$
W(t)=\sum_{w \in W} t^{l(w)}
$$

where $l$ is the length function on $W$. A key result in the theory of reflection groups is the Chevalley-Solomon product formula [11,33.

$$
W(t)=\prod_{i=1}^{r} \frac{1-t^{d_{i}}}{1-t},
$$

where the $d_{1}, \ldots, d_{r} \geq 2$ are the degrees of the fundamental invariants.

For reflection groups of crystallographic type, i.e., Weyl groups, Macdonald [29] generalised the Poincaré polynomial to a multivariable polynomial $W(\boldsymbol{t})$ by attaching a variable $t_{\alpha}$ to each positive root $\alpha$ of the underlying root system. To be more precise, let $R$ be a reduced irreducible root system of rank $r$, and $R^{+}$(resp. $R^{-}=-R^{+}$) the set of positive (resp. negative) roots. Write $\alpha>0$ if $\alpha \in R^{+}$. Let $\boldsymbol{t}$ denote the alphabet $\boldsymbol{t}=\left\{t_{\alpha}\right\}_{\alpha>0}$ and for $S \subseteq R^{+}$define the word $t_{S}=\prod_{\alpha \in S} t_{\alpha}$.

Received by the editors October 5, 2012 and, in revised form, February 27, 2013.

2010 Mathematics Subject Classification. Primary 05A19, 05E05, 17B22, 20F55.

Key words and phrases. Constant term identities, Kadell's conjecture, Poincaré polynomials, polynomial lemma, (0,1)-matrices.

This work was supported by the Australian Research Council and by Hungarian National Scientific Research Funds (OTKA) Grant K100291.

We deeply regret that Alain Lascoux passed away on October 20, 2013. 
Macdonald's multivariable Poincaré polynomial is then given by

$$
W(\boldsymbol{t})=\sum_{w \in W} t_{R(w)},
$$

where $R(w)=R^{+} \cap w\left(R^{-}\right)$. Since $|R(w)|=l(w)$, the multivariable Poincaré polynomial reduces to (1.1) when $t_{\alpha}=t$ for all $\alpha$.

In its full generality $W(\boldsymbol{t})$ no longer admits a product form. Instead Macdonald [29, Theorem 2.8] showed (see also [36, Theorem 1]) that it may be expressed as

$$
W(\boldsymbol{t})=\sum_{w \in W} \prod_{\alpha>0} \frac{1-t_{\alpha} \mathrm{e}^{-w(\alpha)}}{1-\mathrm{e}^{-w(\alpha)}},
$$

with $\mathrm{e}^{\alpha}$ a formal exponential.

A different discovery of Macdonald, formulated as a conjecture, is the constant term identity 30]

$$
\mathrm{CT}\left[\prod_{\alpha>0}\left(\mathrm{e}^{-\alpha}, q \mathrm{e}^{\alpha}\right)_{k}\right]=\prod_{i=1}^{r}\left[\begin{array}{c}
d_{i} k \\
k
\end{array}\right]
$$

where

$$
(a)_{k}=\prod_{i=0}^{k-1}\left(1-a q^{i}\right) \quad \text { and } \quad\left(a_{1}, \ldots, a_{m}\right)_{k}=\left(a_{1}\right)_{k} \cdots\left(a_{m}\right)_{k}
$$

are $q$-shifted factorials and

$$
\left[\begin{array}{c}
n \\
m
\end{array}\right]= \begin{cases}\frac{(q)_{n}}{(q)_{m}(q)_{n-m}} & \text { for } 0 \leq m \leq n \\
0 & \text { otherwise }\end{cases}
$$

is a $q$-binomial coefficient [3, 15. There is a large literature on (1.4) (see e.g., 13] and the references therein), with an ultimately case-free proof found by Cherednik [8,9] based on his double affine Hecke algebra [10].

It is not at all difficult to also express the multivariable Poincaré polynomial as a constant term:

$$
W(\boldsymbol{t})=\mathrm{CT}\left[\prod_{\alpha>0}\left(1-\mathrm{e}^{-\alpha}\right)\left(1-t_{\alpha} \mathrm{e}^{\alpha}\right)\right] .
$$

Since this generalises the $k=1$ case of Macdonald's conjecture, it then takes little to note that (1.4) and (1.5) can in fact be unified.

Proposition 1.1. For $k \geq 1$ we have

$$
\mathrm{CT}\left[\prod_{\alpha>0}\left(1-\mathrm{e}^{-\alpha}\right)\left(1-t_{\alpha} \mathrm{e}^{\alpha}\right)\left(q \mathrm{e}^{-\alpha}, q \mathrm{e}^{\alpha}\right)_{k-1}\right]=W(\boldsymbol{t}) \prod_{i=1}^{r}\left[\begin{array}{c}
d_{i} k-1 \\
k-1
\end{array}\right] .
$$

This is perhaps an elegant result - connecting Poincaré polynomials and Macdonald-type constant term identities - but, assuming (1.4), not at all deep. More interesting is what happens if one restricts considerations to the root system $\mathrm{A}_{n-1}$ for which the set of positive roots may be taken to be $R^{+}=\left\{\epsilon_{i}-\epsilon_{j}: 1 \leq i<\right.$ $j \leq n\}$, with $\epsilon_{i}$ the $i$ th standard unit vector in $\mathbb{R}^{n}$. Then (1.4) admits the following 
inhomogeneous generalisation known as the Andrews' $q$-Dyson conjecture [2] or the Zeilberger-Bressoud theorem [37] (see also [6, 16, 24]):

$$
\mathrm{CT}\left[\prod_{1 \leq i<j \leq n}\left(x_{i} / x_{j}\right)_{a_{i}}\left(q x_{j} / x_{i}\right)_{a_{j}}\right]=\frac{(q)_{a_{1}+\cdots+a_{n}}}{(q)_{a_{1}} \cdots(q)_{a_{n}}}
$$

where we have identified $\exp \left(-\epsilon_{i}\right)$ with $x_{i}$. In view of Proposition 1.1 it is natural to try to generalise (1.7) by replacing

$$
\left(q x_{j} / x_{i}\right)_{a_{j}} \text { by }\left(q x_{j} / x_{i}\right)_{a_{j}-1}\left(1-t_{i j} x_{j} / x_{i}\right) .
$$

Here we have made the further identification of $t_{\epsilon_{i}-\epsilon_{j}}$ with $t_{i j}$. To describe the resulting constant term identity we need some further notation.

Let $\left(\begin{array}{c}{[n]} \\ 2\end{array}\right)=\{(i, j): 1 \leq i<j \leq n\}$ and for $S \subseteq\left(\begin{array}{c}{[n]} \\ 2\end{array}\right)$ set $t_{S}=\prod_{(i, j) \in S} t_{i j}$. Let $I(w)=\left\{(i, j) \in\left(\begin{array}{c}{[n]} \\ 2\end{array}\right): w(i)>w(j)\right\}$ be the inversion set of the permutation $w \in \mathfrak{S}_{n}$ and $R(w)=I\left(w^{-1}\right)=\left\{(w(j), w(i)) \in\left(\begin{array}{c}{[n]} \\ 2\end{array}\right): i<j\right\}$. For $a=\left(a_{1}, \ldots, a_{n}\right)$ a sequence of nonnegative integers we write $|a|$ and $\sigma_{i}$ for the sum of its components and the $i$ th partial sum respectively, i.e., $|a|=a_{1}+\cdots+a_{n}$ and $\sigma_{i}=a_{1}+\cdots+a_{i}$. The notation $|a|$ and $\sigma_{n}$ will be used interchangeably. The $q$-multinomial coefficient $\left[\begin{array}{c}|a| \\ a\end{array}\right]$ can now be defined as

$$
\left[\begin{array}{c}
|a| \\
a
\end{array}\right]=\frac{(q)_{|a|}}{(q)_{a_{1}} \cdots(q)_{a_{n}}}=\prod_{i=1}^{n}\left[\begin{array}{c}
\sigma_{i} \\
a_{i}
\end{array}\right] .
$$

Finally we introduce an inhomogeneous version of the multivariable $\mathrm{A}_{n-1}$ Poincaré polynomial, with coefficients in $\mathbb{Q}(q)$, as follows:

$$
W_{a}(\boldsymbol{t})=\sum_{w \in \mathfrak{S}_{n}} t_{R(w)} \prod_{i=1}^{n} \frac{1-q^{\sigma_{i}}}{1-q^{w\left(\sigma_{i}\right)}}
$$

where $w\left(\sigma_{i}\right)=a_{w(1)}+\cdots+a_{w(i)}$. Note that $W_{(k, \ldots, k)}(\boldsymbol{t})=W(\boldsymbol{t})$.

Theorem 1.2. Let $a=\left(a_{1}, \ldots, a_{n}\right)$ be a sequence of positive integers. Then

$$
\mathrm{CT}\left[\prod_{1 \leq i<j \leq n}\left(x_{i} / x_{j}\right)_{a_{i}}\left(q x_{j} / x_{i}\right)_{a_{j}-1}\left(1-t_{i j} x_{j} / x_{i}\right)\right]=W_{a}(\boldsymbol{t}) \prod_{i=1}^{n}\left[\begin{array}{c}
\sigma_{i}-1 \\
a_{i}-1
\end{array}\right] .
$$

For $a_{1}=\cdots=a_{n}=k$ the right-hand side simplifies to

$$
W(\boldsymbol{t}) \prod_{i=1}^{n-1}\left[\begin{array}{c}
(i+1) k-1 \\
k-1
\end{array}\right]
$$

and we recover the $\mathrm{A}_{n-1}$ case of (1.6).

Our proof of Theorem 1.2 uses the polynomial lemma of Lason [27] and KarasevPetrov [22] - a form of multivariable Lagrange interpolation in the spirit of the Combinatorial Nullstellensatz [1. The efficacy of the polynomial lemma to constant term identities was recently demonstrated in [24] in the form of a one-page proof of the $q$-Dyson conjecture (1.7).

Several constant term identities due to Bressoud and Goulden [6] follow from Theorem 1.2 in a very easy manner. If $t_{i j}=0$ for all $i$ and $j$, then $W_{a}(\boldsymbol{t})=1$ and we obtain [6, Theorem 2.2, $\sigma=\mathrm{id}]$. More generally, for $I \subseteq\{1,2, \ldots, n\}$ and $\bar{I}$ its complement, let $t_{i j}=0$ if $j \in I$ and $t_{i j}=q^{a_{j}}$ if $j \in \bar{I}$. Then only those permutations 
$w$ contribute to $W_{a}(\boldsymbol{t})$ for which $w(i)=i$ for $i \in I$. Replacing the sequence $\left(q^{a_{j}}\right)_{j \in \bar{I}}$ by $\left(u_{1}, \ldots, u_{m}\right)(m:=|\bar{I}|)$, we are left with the simple computation (see page 6828)

$$
\sum_{w \in \mathfrak{S}_{m}} w\left(\prod_{i=1}^{m} \frac{1-u_{i}}{1-u_{1} \cdots u_{i}}\right) \prod_{(i, j) \in R(w)} u_{j}=1 .
$$

Hence $W_{a}(\boldsymbol{t})=\prod_{i \in \bar{I}}\left(1-q^{\sigma_{i}}\right) /\left(1-q^{a_{i}}\right)$, and we obtain the generalised $q$-Dyson identity [6, Theorem 2.5]

$$
\mathrm{CT}\left[\prod_{1 \leq i<j \leq n}\left(x_{i} / x_{j}\right)_{a_{i}}\left(q x_{j} / x_{i}\right)_{a_{j}-\chi(j \in I)}\right]=\left[\begin{array}{c}
|a| \\
a
\end{array}\right] \prod_{i \in I} \frac{1-q^{a_{i}}}{1-q^{\sigma_{i}}},
$$

where $\chi$ is the indicator function. Similarly, if $t_{i j}=-1$ we may use

$$
\sum_{w \in \mathfrak{S}_{n}}(-1)^{l(w)} w\left(\prod_{i=1}^{n} \frac{1-u_{i}}{1-u_{1} \cdots u_{i}}\right)=\prod_{1 \leq i<j \leq n} \frac{u_{i}-u_{j}}{1-u_{i} u_{j}}
$$

to find $W_{a}(\boldsymbol{t})=\prod_{i=1}^{n}\left(1-q^{\sigma_{i}}\right) /\left(1-q^{a_{i}}\right) \prod_{i<j}\left(q^{a_{i}}-q^{a_{j}}\right) /\left(1-q^{a_{i}+a_{j}}\right)$. This results in [6. Theorem 2.7]

$$
\mathrm{CT}\left[\prod_{1 \leq i<j \leq n}\left(x_{j} / x_{i}-x_{i} / x_{j}\right) \prod_{i \neq j}\left(q x_{i} / x_{j}\right)_{a_{i}-1}\right]=\left[\begin{array}{c}
|a| \\
a
\end{array}\right] \prod_{1 \leq i<j \leq n} \frac{q^{a_{i}}-q^{a_{j}}}{1-q^{a_{i}+a_{j}}} .
$$

The fact that Theorem 1.2 allows us to reprove the constant term identities of Bressoud and Goulden is not too surprising. Combining two of the key theorems of their paper - both formulated in the language of tournaments - and reinterpreting these in terms of permutations provides an alternative method of proof of Theorem 1.2 .

As a much deeper and more interesting application than the reproof of known results, we will show that Theorem 1.2 may be used to prove Kadell's $q$-Dyson orthogonality conjecture 21].

For $x=\left(x_{1}, \ldots, x_{n}\right)$ and $u=\left(u_{1}, \ldots, u_{n}\right)$ a sequence of integers, write $x^{u}$ for the monomial $x_{1}^{u_{1}} \cdots x_{n}^{u_{n}}$. If all $u_{i}$ are nonnegative we refer to $u$ as a composition and, if in addition $u_{1}+\cdots+u_{n}=k$, we write $|u|=k$. The set of all compositions of the form $\left(u_{1}, \ldots, u_{n}\right)$ will be denoted by $\mathscr{C}_{n}$. If $u \in \mathscr{C}_{n}$ satisfies $u_{1} \geq u_{2} \geq \cdots \geq u_{n}$ we say that $u$ is a partition, and $\mathscr{P}_{n}$ will denote the set of all partitions in $\mathscr{C}_{n}$. As is customary, we will often denote partitions by the Greek letters $\lambda, \mu, \nu$ and not display their tails of zeros. The unique partition in the $\mathfrak{S}_{n}$ orbit of $u \in \mathscr{C}_{n}$ is denoted by $u^{+}$. Let $s_{\lambda}(x)$ be the classical Schur function [31]

$$
s_{\lambda}(x)=\frac{\operatorname{det}_{1 \leq i, j \leq n}\left(x_{i}^{\lambda_{j}+n-j}\right)}{\prod_{1 \leq i<j \leq n}\left(x_{i}-x_{j}\right)}
$$

for $\lambda \in \mathscr{P}_{n}$. For $\lambda=(m)$ this simplifies to the $m$ th complete symmetric function

$$
h_{m}(x)=\sum_{|v|=m} x^{v}
$$

Given a sequence of nonnegative integers $a=\left(a_{1}, \ldots, a_{n}\right)$ let

$$
x^{(a)}=\left(x_{1}, x_{1} q, \ldots, x_{1} q^{a_{1}-1}, \ldots, x_{n}, x_{n} q, \ldots, x_{n} q^{a_{n}-1}\right)
$$

or, in the notation of $\lambda$-rings [25],

$$
x^{(a)}=x_{1} \frac{1-q^{a_{1}}}{1-q}+\cdots+x_{n} \frac{1-q^{a_{n}}}{1-q} .
$$


We will be interested in constant terms of the form

$$
D_{v, \lambda}(a):=\mathrm{CT}\left[x^{-v} s_{\lambda}\left(x^{(a)}\right) \prod_{1 \leq i<j \leq n}\left(x_{i} / x_{j}\right)_{a_{i}}\left(q x_{j} / x_{i}\right)_{a_{j}}\right]
$$

for $v \in \mathscr{C}_{n}$ and $\lambda \in \mathscr{P}_{|a|}$. Before stating Kadell's conjecture we make a few general comments about the above constant term. First, by homogeneity $D_{v, \lambda}(a)=0$ if $|v| \neq|\lambda|$. Second, if $n=1$, and viewing $v$ and $a$ as scalars,

$$
D_{v, \lambda}(a)=s_{\lambda}\left(\frac{1-q^{a}}{1-q}\right) \operatorname{CT}\left[x^{|\lambda|-v}\right]=\delta_{v,|\lambda|} q^{\sum_{i<j} \lambda_{j}} \prod_{s \in \lambda} \frac{1-q^{a+c(s)}}{1-q^{h(s)}},
$$

where $c(s)$ and $h(s)$ are the content and hook-length of the square $s$ in the diagram of $\lambda$; see [31, p. 44]. As a third remark we note that if $a_{k}=0$, then $s_{\lambda}\left(x^{(a)}\right)$ does not depend on $x_{k}$, and the double product over $i<j$, viewed as a function of $x_{k}$, takes the form $c_{0}+c_{-1} x_{k}^{-1}+c_{-2} x_{k}^{-2}+\cdots$. Hence the constant term will be zero unless $v_{k}=0$. Lastly, it is natural to more generally consider (1.12) for $v$ an arbitrary element of $\mathbb{Z}^{n}$. Unfortunately, the method developed for proving Kadell's orthogonality conjecture has little to say about this more general range of $v$. As we shall see later, the $i$ th entry of the composition $v$ arises as the $i$ th row sum of a $(0,1)$-matrix. Only in Section [5 will we consider (1.12) for $v \notin \mathscr{C}_{n}$.

Theorem 1.3 (Kadell's orthogonality conjecture [21]). For $m$ a positive integer, $v \in \mathscr{C}_{n}$ and $a=\left(a_{1}, \ldots, a_{n}\right)$ a sequence of nonnegative integers,

$$
D_{v,(m)}(a)=0 \quad \text { if } v^{+} \neq(m)
$$

and

$$
D_{v,(m)}(a)=\frac{q^{\sigma_{n}-\sigma_{k}}\left(1-q^{a_{k}}\right)\left(q^{|a|}\right)_{m}}{\left(1-q^{|a|}\right)\left(q^{|a|-a_{k}+1}\right)_{m}}\left[\begin{array}{c}
|a| \\
a
\end{array}\right] \quad \text { if } v=(\underbrace{0, \ldots, 0}_{k-1 \text { times }}, m, \underbrace{0, \ldots, 0}_{n-k \text { times }}) .
$$

We remark that Kadell's original conjecture only includes the $k=1$ case of (1.13b), i.e., $v=(m)$, and misses both the term $q^{\sigma_{n}-\sigma_{1}}$ and the +1 in $\left(q^{|a|-a_{1}+1}\right)_{m}$.

We obtain several more general results than Theorem 1.3 involving Schur functions. A particularly simple example is

$$
D_{\lambda, \lambda}(a)=q^{\sum_{i<j} a_{j}} \prod_{i=1}^{n}\left[\begin{array}{c}
\lambda_{i}+a_{i}+\cdots+a_{n}-1 \\
a_{i}-1
\end{array}\right],
$$

provided $\lambda$ is a strict partition, i.e., $\lambda_{1}>\lambda_{2}>\cdots>\lambda_{n} \geq 0$ and all $a_{i}>0$.

The remainder of this paper is organised as follows. In the next section we give a simple proof of an inhomogeneous version of Proposition 1.1. Then, in Section 3 , we use the polynomial lemma to give a proof of Theorem 1.2 and show how the theorem relates to constant term identities of Bressoud and Goulden. In Section 4 , we apply Theorem 1.2 to prove and generalise Kadell's orthogonality conjecture. Finally, in Section [5] answering a question raised by the anonymous referee, we show that Kadell's orthogonality conjecture implies a conjecture of Sills 32 proved previously by Lv, Xin and Zhou using different means 28]. 


\section{Proposition 1.1 And its Inhomogeneous EXtension}

This section, which is elementary in its contents, may be viewed as a warm-up exercise to the more involved considerations of subsequent sections. We do however assume the reader has a basic knowledge of root systems; see e.g., 5, 17.

Although (1.5) is a special case of Proposition 1.1] we establish it prior to proving the more general result. We make this distinction because the former requires little more than the Weyl denominator formula, whereas our proof of Proposition 1.1 relies on the deep result (1.4).

Let $\rho=(1 / 2) \sum_{\alpha>0} \alpha$ be the Weyl vector of $R$. By the Weyl denominator formula

$$
\prod_{\alpha>0}\left(1-\mathrm{e}^{-\alpha}\right)=\sum_{w \in W}(-1)^{l(w)} \mathrm{e}^{w(\rho)-\rho}
$$

and the expansion

$$
\prod_{\alpha>0}\left(1-t_{\alpha} \mathrm{e}^{\alpha}\right)=\sum_{S \subseteq R^{+}}(-1)^{|S|} t_{S} \mathrm{e}^{\sum_{\alpha \in S} \alpha}
$$

we have

$$
\mathrm{CT}\left[\prod_{\alpha>0}\left(1-\mathrm{e}^{-\alpha}\right)\left(1-t_{\alpha} \mathrm{e}^{\alpha}\right)\right]=\sum_{w \in W} \sum_{S \subseteq R^{+}}(-1)^{l(w)+|S|} t_{S} \mathrm{CT}\left[\mathrm{e}^{w(\rho)-\rho+\sum_{\alpha \in S} \alpha}\right] .
$$

Since $w(\rho)-\rho=-\sum_{\alpha \in R(w)} \alpha$ (see, e.g., [29, p. 167]) the right-hand side may also be written as

$$
\sum_{w \in W} \sum_{S \subseteq R^{+}}(-1)^{l(w)+|S|} t_{S} \mathrm{CT}\left[\mathrm{e}^{\sum_{\alpha \in S} \alpha-\sum_{\alpha \in R(w)} \alpha}\right]
$$

The constant term vanishes unless $S=R(w) \subseteq R^{+}$so that we are left with $\sum_{w \in W} t_{R(w)}=W(\boldsymbol{t})$, as claimed.

Next we turn to the proof of the more general Proposition 1.1. In fact, what we shall prove is an inhomogeneous version of the proposition which generalises another ex-conjecture of Macdonald, also proved by Cherednik. Let $\left\{k_{\alpha}\right\}_{\alpha>0}$ be a set of integers constant along Weyl orbits, i.e., $k_{\alpha}=k_{\beta}$ for $\|\alpha\|=\|\beta\|$. Then [30, Conjecture 2.3], [8, Theorem 1.1], [9, Theorem 0.1]

$$
\mathrm{CT}\left[\prod_{\alpha>0}\left(\mathrm{e}^{-\alpha}, q \mathrm{e}^{\alpha}\right)_{k_{\alpha}}\right]=\prod_{\alpha>0} \frac{(q)_{\left(\rho_{k}, \alpha^{\vee}\right)+k_{\alpha}}(q)_{\left(\rho_{k}, \alpha^{\vee}\right)-k_{\alpha}}}{(q)_{\left(\rho_{k}, \alpha^{\vee}\right)}^{2}}
$$

where $\rho_{k}:=\frac{1}{2} \sum_{\alpha>0} k_{\alpha} \alpha,(\cdot, \cdot)$ is the standard symmetric bilinear form on $R$ and $\alpha^{\vee}=2 \alpha /(\alpha, \alpha)$ a coroot.

Proposition 2.1. For positive integers $k_{\alpha}$, constant along Weyl orbits,

$$
\begin{aligned}
\mathrm{CT}\left[\prod_{\alpha>0}\left(1-\mathrm{e}^{-\alpha}\right)\left(1-t_{\alpha} \mathrm{e}^{\alpha}\right)\left(q \mathrm{e}^{-\alpha}, q \mathrm{e}^{\alpha}\right)_{k_{\alpha}-1}\right] \\
\quad=W(\boldsymbol{t}) \prod_{\alpha>0} \frac{(q)_{\left(\rho_{k}, \alpha^{\vee}\right)+k_{\alpha}-1}(q)_{\left(\rho_{k}, \alpha^{\vee}\right)-k_{\alpha}}}{(q)_{\left(\rho_{k}, \alpha^{\vee}\right)-1}(q)_{\left(\rho_{k}, \alpha^{\vee}\right)}} .
\end{aligned}
$$


Proof. If we apply [9, Lemma 4.4] with $f$ therein chosen as $\prod_{\alpha>0}\left(\mathrm{e}^{-\alpha}, \mathrm{e}^{\alpha}\right)_{k_{\alpha}}$ and $k_{\alpha}>0$, then

$$
\mathrm{CT}\left[\prod_{\alpha>0}\left(\mathrm{e}^{-\alpha}, q \mathrm{e}^{\alpha}\right)_{k_{\alpha}}\right]=\frac{1}{|W|} \prod_{\alpha>0} \frac{1-q^{\left(\rho_{k}, \alpha^{\vee}\right)+k_{\alpha}}}{1-q^{\left(\rho_{k}, \alpha^{\vee}\right)}} \cdot \mathrm{CT}\left[\prod_{\alpha>0}\left(\mathrm{e}^{-\alpha}, \mathrm{e}^{\alpha}\right)_{k_{\alpha}}\right],
$$

where $|W|=d_{1} \cdots d_{r}$ is the order of $W$. Hence (2.2) for $k_{\alpha}>0$ may be rewritten as

$$
\mathrm{CT}\left[\prod_{\alpha>0}\left(\mathrm{e}^{-\alpha}, \mathrm{e}^{\alpha}\right)_{k_{\alpha}}\right]=|W| \prod_{\alpha>0} \frac{(q)_{\left(\rho_{k}, \alpha^{\vee}\right)+k_{\alpha}-1}(q)_{\left(\rho_{k}, \alpha^{\vee}\right)-k_{\alpha}}}{(q)_{\left(\rho_{k}, \alpha^{\vee}\right)-1}(q)_{\left(\rho_{k}, \alpha^{\vee}\right)}} .
$$

This is of course the $t_{\alpha}=1$ case of (2.3).

Now abbreviate the left-hand side of (2.3) by CT[... . Then

$$
\mathrm{CT}[\ldots]=\mathrm{CT}\left[\prod_{\alpha>0}\left(\mathrm{e}^{-\alpha}, \mathrm{e}^{\alpha}\right)_{k_{\alpha}} \frac{1-t_{\alpha} \mathrm{e}^{\alpha}}{1-\mathrm{e}^{\alpha}}\right]=\mathrm{CT}\left[\prod_{\alpha>0}\left(\mathrm{e}^{-\alpha}, \mathrm{e}^{\alpha}\right)_{k_{\alpha}} \frac{1-t_{\alpha} \mathrm{e}^{-\alpha}}{1-\mathrm{e}^{-\alpha}}\right] .
$$

Since acting on the above kernel with the Weyl group does not affect the constant term, this is also

$$
\mathrm{CT}[\ldots]=\frac{1}{|W|} \mathrm{CT}\left[\prod_{\alpha>0}\left(\mathrm{e}^{-\alpha}, \mathrm{e}^{\alpha}\right)_{k_{\alpha}} \cdot \sum_{w \in W} \prod_{\alpha>0} \frac{1-t_{\alpha} \mathrm{e}^{-w(\alpha)}}{1-\mathrm{e}^{-w(\alpha)}}\right] .
$$

By Macdonald's formula (1.3) the sum over $W$ yields $W(\boldsymbol{t})$ so that

$$
\mathrm{CT}[\ldots]=\frac{W(\boldsymbol{t})}{|W|} \mathrm{CT}\left[\prod_{\alpha>0}\left(\mathrm{e}^{-\alpha}, \mathrm{e}^{\alpha}\right)_{k_{\alpha}}\right] .
$$

Thanks to (2.4) the proof is done.

We note that if we set $t_{\alpha}=q^{k_{\alpha}}$ in (2.3), then the constant term on the left coincides with the constant term in (2.2). Using the latter identity we thus infer that

$$
\left.W(\boldsymbol{t})\right|_{t_{\alpha}=q^{k_{\alpha}}}=\prod_{\alpha>0} \frac{1-q^{\left(\rho_{k}, \alpha^{\vee}\right)+k_{\alpha}}}{1-q^{\left(\rho_{k}, \alpha^{\vee}\right)}} .
$$

Since this is a rational function identity (polynomial in fact), $q^{k_{\alpha}}$ may be replaced by $t_{\alpha}$ resulting in

$$
\left.W(\boldsymbol{t})\right|_{t_{\alpha} \text { constant along } W \text {-orbits }}=\prod_{\alpha>0} \frac{1-t_{\alpha} \prod_{\beta>0} t_{\beta}^{\left(\beta, \alpha^{\vee}\right) / 2}}{1-\prod_{\beta>0} t_{\beta}^{\left(\beta, \alpha^{\vee}\right) / 2}} .
$$

Curiously, this product form for a restricted version of the multivariable Poincaré polynomial is slightly different from the one given by Macdonald in 29, Theorem $2.4]$ :

$$
\left.W(\boldsymbol{t})\right|_{t_{\alpha} \text { constant along } W \text {-orbits }}=\prod_{\alpha>0} \frac{1-t_{\alpha} \prod_{\beta>0} t_{\beta}^{\left(\alpha, \beta^{\vee}\right) / 2}}{1-\prod_{\beta>0} t_{\beta}^{\left(\alpha, \beta^{\vee}\right) / 2}},
$$

although equality of the above two products is readily established. 


\section{THEOREM 1.2}

In this section we give a proof Theorem 1.2 following the method of the recent proof of the $q$-Dyson conjecture given in [24]. We also present a reformulation of the theorem in terms of tournaments, thus connecting the theorem with results of Bressoud and Goulden.

\subsection{Proof of Theorem 1.2, Write}

$$
\mathrm{CT}\left[\prod_{1 \leq i<j \leq n}\left(x_{i} / x_{j}\right)_{a_{i}}\left(q x_{j} / x_{i}\right)_{a_{j}-1}\left(1-t_{i j} x_{j} / x_{i}\right)\right]=\sum_{S \subseteq\left(\begin{array}{c}
{[n]} \\
2
\end{array}\right)} \mathrm{c}(a ; S) t_{S},
$$

where the coefficients c $(a ; S)$ are independent of the $t_{i j}$. Thus, c $(a ; S)$ is the coefficient of $\prod_{(i, j) \in S}\left(x_{i} / x_{j}\right)$ in the Laurent polynomial

$$
(-1)^{|S|} \prod_{1 \leq i<j \leq n}\left(x_{i} / x_{j}\right)_{a_{i}}\left(q x_{j} / x_{i}\right)_{a_{j}-1}
$$

which (recalling the abbreviation $|a|=a_{1}+\cdots+a_{n}$ ) is the same as the coefficient of the monomial

$$
\prod_{(i, j) \in S}\left(x_{i} / x_{j}\right) \cdot \prod_{i=1}^{n} x_{i}^{|a|-a_{i}-(n-i)}
$$

in the homogeneous polynomial

$$
F_{S}(x)=(-1)^{|S|} \prod_{1 \leq i<j \leq n}\left(\prod_{k=0}^{a_{i}-1}\left(x_{j}-x_{i} q^{k}\right) \cdot \prod_{k=1}^{a_{j}-1}\left(x_{i}-x_{j} q^{k}\right)\right) .
$$

Although the only dependence on $S$ is through the factor $(-1)^{|S|}$, it has been included to simplify subsequent equations.

To express this coefficient we apply the following simple consequence of multivariate Lagrange interpolation, independently formulated by Lason [27] and by Karasev and Petrov 22].

Lemma 3.1. Let $\mathbb{F}$ be an arbitrary field and $F \in \mathbb{F}\left[x_{1}, x_{2}, \ldots, x_{n}\right]$ a polynomial of degree $\operatorname{deg}(F) \leq d_{1}+d_{2}+\cdots+d_{n}$. For arbitrary subsets $A_{1}, A_{2}, \ldots, A_{n}$ of $\mathbb{F}$ with $\left|A_{i}\right|=d_{i}+1$, the coefficient of $\prod x_{i}^{d_{i}}$ in $F$ is

$$
\sum_{c_{1} \in A_{1}} \sum_{c_{2} \in A_{2}} \cdots \sum_{c_{n} \in A_{n}} \frac{F\left(c_{1}, c_{2}, \ldots, c_{n}\right)}{\phi_{1}^{\prime}\left(c_{1}\right) \phi_{2}^{\prime}\left(c_{2}\right) \cdots \phi_{n}^{\prime}\left(c_{n}\right)},
$$

where $\phi_{i}(z)=\prod_{a \in A_{i}}(z-a)$.

The idea is to apply this lemma with $\mathbb{F}=\mathbb{Q}(q)$ and $F=F_{S}$ and with a suitable choice of the sets $A_{i}$ such that $F_{S}(c) \neq 0$ holds for at most one element $c \in$ $A_{1} \times \cdots \times A_{n}$. This will allow us to express the coefficient c $(a ; S)$ in a simple product form if there is an element $w \in \mathfrak{S}_{n}$ such that $S=R(w)$ or else to prove that $\mathrm{c}(a ; S)=0$. Note that

$$
\prod_{(i, j) \in S}\left(x_{i} / x_{j}\right) \cdot \prod_{i=1}^{n} x_{i}^{|a|-a_{i}-(n-i)}=\prod_{i=1}^{n} x_{i}^{|a|-a_{i}-(n-i)-d_{i}+e_{i}},
$$


where $d_{j}=|\{i:(i, j) \in S\}|$ and $e_{i}=|\{j:(i, j) \in S\}|$. Clearly $d_{i} \leq i-1$, $e_{i} \leq n-i$, and, recalling that each $a_{i}>0$, we therefore have

$$
0 \leq|a|-a_{i}-(n-i)-d_{i}+e_{i} \leq|a|-a_{i} .
$$

Thus, there exist sets $B_{i} \subseteq\left\{0,1, \ldots,|a|-a_{i}\right\}$ such that

$$
\left|B_{i}\right|=|a|-a_{i}-(n-i)-d_{i}+e_{i}+1 .
$$

We will construct the $A_{i}$ in the form

$$
A_{i}=\left\{q^{\alpha_{i}}: \alpha_{i} \in B_{i}\right\} \subset \mathbb{F}=\mathbb{Q}(q)
$$

with an appropriate choice of the sets $B_{i}$. Before specifying these sets further, we first note that they have the right cardinality for a possible application of Lemma 3.1 .

Assume that $c_{i}=q^{\alpha_{i}} \in A_{i}$ and $F_{S}(c) \neq 0$. Then all $\alpha_{i}$ are distinct. Moreover, $\alpha_{i} \geq \alpha_{j}+a_{j}$ holds for $\alpha_{i}>\alpha_{j}$. Consider the unique permutation $\pi \in \mathfrak{S}_{n}$ for which

$$
0 \leq \alpha_{\pi(1)}<\alpha_{\pi(2)}<\cdots<\alpha_{\pi(n)} \leq|a|-a_{\pi(n)} .
$$

Given that

$$
|a|-a_{\pi(n)}=\sum_{i=1}^{n-1} a_{\pi(i)} \leq \sum_{i=1}^{n-1}\left(\alpha_{\pi(i+1)}-\alpha_{\pi(i)}\right)=\alpha_{\pi(n)}-\alpha_{\pi(1)} \leq|a|-a_{\pi(n)},
$$

it follows that $\alpha_{\pi(i)}=a_{\pi(1)}+\cdots+a_{\pi(i-1)}$ holds for every $1 \leq i \leq n$.

Consider $\ell_{i}:=(n-i)+d_{i}-e_{i}$. Then $0 \leq \ell_{i} \leq n-1$. Denote by $K=K(S)$ the smallest nonnegative integer that does not occur exactly once among the numbers $\ell_{1}, \ldots, \ell_{n}$. More formally, $K$ is the largest nonnegative integer $k$ for which

$$
\left|\left\{i: \ell_{i}=j\right\}\right|=1
$$

holds for every integer $0 \leq j<k$. Such a $K \leq n$ clearly exists. Our construction of the appropriate sets $B_{i}$ (and hence $A_{i}$ ) relies on the following lemma, stating that $K$ is not among the $\ell_{i}$.

Lemma 3.2. $\left|\left\{i: \ell_{i}=K\right\}\right|=0$.

Proof. The proof is by induction on $n$. For the initial step $n=2$ we have $\ell_{2}=0$, $\ell_{1}=1$ if $S=\emptyset$ and $\ell_{1}=0, \ell_{2}=1$ if $S=\{(1,2)\}$. In both cases $K=2$ and the conclusion trivially holds. So assume that $n \geq 3$ and the statement has been proven for smaller values of $n$. First consider the case $K=0$, meaning $\left|\left\{i: \ell_{i}=0\right\}\right| \neq 1$. It is enough to show that $\left|\left\{i: \ell_{i}=0\right\}\right|<2$. Suppose that, on the contrary, $\ell_{i}=\ell_{j}=0$ holds for some $1 \leq i<j \leq n$. This means that $d_{i}=d_{j}=0, e_{i}=n-i$ and $e_{j}=n-j$. However, if $(i, j) \in S$, then $d_{j}>0$, whereas if $(i, j) \notin S$, then $e_{i}<n-i$, a contradiction.

Turning to the general case $K>0$, by definition there is a unique $1 \leq \alpha \leq n$ such that $\ell_{\alpha}=0$, whence $d_{\alpha}=0$ and $e_{\alpha}=n-\alpha$. Construct $S^{\prime} \subseteq\left(\begin{array}{c}{[n-1]} \\ 2\end{array}\right)$ induced by $S$ as follows. First, for $1 \leq i<j<\alpha$ let $(i, j) \in S^{\prime}$ if and only if $(i, j) \in S$. Next, for $1 \leq i<\alpha \leq j \leq n-1$ let $(i, j) \in S^{\prime}$ if and only if $(i, j+1) \in S$. Finally, for $\alpha \leq i<j \leq n-1$ let $(i, j) \in S^{\prime}$ if and only if $(i+1, j+1) \in S$. For this new set $S^{\prime}$ we have

$$
d_{j}^{\prime}=\left|\left\{i:(i, j) \in S^{\prime}\right\}\right|=d_{j+\chi(j \geq \alpha)}-\chi(j \geq \alpha)
$$


and

$$
e_{i}^{\prime}=\left|\left\{j:(i, j) \in S^{\prime}\right\}\right|=e_{i+\chi(i \geq \alpha)},
$$

where the indicator $\chi(\mathcal{T})$ is 1 if $\mathcal{T}$ is true and 0 otherwise. Consequently,

$$
\ell_{i}^{\prime}=((n-1)-i)+d_{i}^{\prime}-e_{i}^{\prime}=\ell_{i+\chi(i \geq \alpha)}-1
$$

and

$$
K^{\prime}=\max \left\{k \geq 0:\left|\left\{i: \ell_{i}^{\prime}=j\right\}\right|=1 \text { for every } 0 \leq j<k\right\}=K-1 \leq n-1 .
$$

It follows from the induction hypothesis that $\left|\left\{i: \ell_{i}^{\prime}=K^{\prime}\right\}\right|=0$. Since $\ell_{\alpha}=0 \neq K$, this is equivalent to the statement $\left|\left\{i: \ell_{i}=K\right\}\right|=0$.

Now define the sets $B_{i}$ satisfying $B_{i} \subseteq\left\{0,1, \ldots,|a|-a_{i}\right\}$ and $\left|B_{i}\right|=|a|-a_{i}-\ell_{i}+1$ as follows. For $k=1,2, \ldots, K$ write $\tau(k)$ for the unique integer $i$ for which $\ell_{i}=k-1$. Let $B_{\tau(1)}=\left\{0,1, \ldots,|a|-a_{\tau(1)}\right\}$. For $2 \leq i \leq K$ let

$$
B_{\tau(i)}=\left\{0,1, \ldots,|a|-a_{\tau(i)}\right\} \backslash\left\{|a|-a_{\tau(i)}-\sum_{j=1}^{k} a_{\tau(j)}: 0 \leq k \leq i-2\right\} .
$$

Finally, if $i \notin\{\tau(1), \ldots, \tau(K)\}$, then $\ell_{i} \geq K+1$ is implied by Lemma 3.2 and therefore we may choose $B_{i}$ as an arbitrary $\left(|a|-a_{i}-\ell_{i}+1\right)$-element subset of

$$
\left\{0,1, \ldots,|a|-a_{\tau(i)}\right\} \backslash\left\{|a|-a_{\tau(i)}-\sum_{j=1}^{k} a_{\tau(j)}: 0 \leq k \leq K\right\} .
$$

Sets $B_{i}$ thus defined, now consider $A_{i}=\left\{q^{\alpha_{i}}: \alpha_{i} \in B_{i}\right\}$. Assume that $c_{i}=q^{\alpha_{i}} \in$ $A_{i}$ and $F_{S}(c) \neq 0$. As we have seen, for such nonvanishing $c$ there is a unique permutation $\pi=\pi_{c} \in \mathfrak{S}_{n}$ such that

$$
\alpha_{\pi(i)}=a_{\pi(1)}+\cdots+a_{\pi(i-1)}=|a|-a_{\pi(i)}-a_{\pi(i+1)}-\cdots-a_{\pi(n)}
$$

holds for every $1 \leq i \leq n$. We will show that such a $c$ exists if and only if $K=n$ and $\pi(i)=\tau(n-i+1)$ for every $1 \leq i \leq n$. First of all, given that $\alpha_{\pi(n)}=|a|-a_{\pi(n)} \in B_{\pi(n)}$, it follows that $K \geq 1$ and $\pi(n)=\tau(1)$. Suppose that for some $1 \leq k<n$ it has been already verified that $K \geq k$ and that $\pi(n-i+1)=\tau(i)$ holds for every $1 \leq i \leq k$. Consider

$\alpha_{\pi(n-k)}=|a|-a_{\pi(n-k)}-a_{\pi(n-k+1)}-\cdots-a_{\pi(n)}=|a|-a_{\pi(n-k)}-a_{\tau(1)}-\cdots-a_{\tau(k)}$.

Given that $\alpha_{\pi(n-k)} \in B_{\pi(n-k)}$, it follows that $K \geq k+1$ and $\pi((n+1)-(k+1))=$ $\pi(n-k)=\tau(k+1)$. Thus our claim follows by induction on $k$.

In summary, $K=K(S)<n$ implies $F(c)=0$ for all $c \in A_{1} \times \cdots \times A_{n}$. In view of Lemma 3.1 it is immediate that $\mathrm{c}(a ; S)=0$ unless $K=n$. In the latter case there is a unique $c \in A_{1} \times \cdots \times A_{n}$ for which $F_{S}(c) \neq 0$, to which there corresponds a unique permutation $\pi=\pi_{c}=\pi_{S} \in \mathfrak{S}_{n}$. First we show that $S=R(w)$ for a suitable element $w \in \mathfrak{S}_{n}$.

Lemma 3.3. Suppose that $K=K(S)=n$ holds for a set $S \subseteq\left(\begin{array}{c}{[n]} \\ 2\end{array}\right)$. Then there exists a permutation $w \in \mathfrak{S}_{n}$ such that $S=R(w)$. Conversely, for any permutation $w \in \mathfrak{S}_{n}$, the set $S=R(w)$ satisfies $K=n$ with $\ell_{w(j)}=n-j$. 
Proof. We prove the first statement by induction on $n$. Recall that $K=n$ holds if and only if $\left\{\ell_{1}, \ell_{2}, \ldots, \ell_{n}\right\}=\{0,1, \ldots, n-1\}$. The initial case $n=2$ is obvious: $\ell_{1}=0, \ell_{2}=1$ if and only if $S=R(21)$, whereas $\ell_{2}=0, \ell_{1}=1$ if and only if $S=R(12)$. Next assume that $n \geq 3$ and that we have already established the claim for smaller values of $n$. Let $\tau(1)=\alpha$ and construct the set $S^{\prime} \subseteq\left(\begin{array}{c}{[n-1]} \\ 2\end{array}\right)$ as in the proof of Lemma 3.2. Then $K^{\prime}=K\left(S^{\prime}\right)=n-1$ and $\left\{\ell_{1}^{\prime}, \ldots, \ell_{n-1}^{\prime}\right\}=\{0, \ldots, n-2\}$, so there exists a permutation $w^{\prime} \in \mathfrak{S}_{n-1}$ such that $S^{\prime}=R\left(w^{\prime}\right)$. To construct $w \in \mathfrak{S}_{n}$, let $w(n)=\alpha$ and $w(i)=w^{\prime}(i)+\chi\left(w^{\prime}(i) \geq \alpha\right)$. It is easy to check that $S=R(w)$.

For the identity permutation $w=$ id we have $S=R(w)=\emptyset, d_{j}=e_{j}=0$, and thus $\ell_{w(j)}=\ell_{j}=n-j$ for every $1 \leq j \leq n$. So to verify the converse statement it is enough to show that if it is true for some permutation $w \in \mathfrak{S}_{n}$, then it also holds for the permutation $w^{\prime}$ obtained from $w$ by the transposition of $w(i)$ and $w(i+1)$ for some $1 \leq i \leq n-1$. Obviously, $\ell_{w^{\prime}(j)}^{\prime}=\ell_{w(j)}^{\prime}=\ell_{w(j)}=n-j$ holds for $j \notin\{i, i+1\}$. Next we verify $\ell_{w^{\prime}(i)}^{\prime}=n-i$. If $w(i)<w(i+1)$, then indeed

$$
\begin{aligned}
\ell_{w^{\prime}(i)}^{\prime} & =\left(n-w^{\prime}(i)\right)+d_{w^{\prime}(i)}^{\prime}-e_{w^{\prime}(i)}^{\prime} \\
& =(n-w(i+1))+\left(d_{w(i+1)}+1\right)-e_{w(i+1)} \\
& =\ell_{w(i+1)}+1 \\
& =n-(i+1)+1 .
\end{aligned}
$$

A similar argument works for the case $w(i)>w(i+1)$, which we leave to the reader along with the verification of $\ell_{w^{\prime}(i+1)}^{\prime}=n-i-1$.

Consider $\mathcal{S}:=\left\{S \subseteq\left(\begin{array}{c}{[n]} \\ 2\end{array}\right): K(S)=n\right\}$. It follows from Lemma 3.3 that the map $w \rightarrow R(w)$ defines a bijection from $\mathfrak{S}_{n}$ to $\mathcal{S}$. In view of $\ell_{\tau(k)}=k-1$ and $\pi(n-i+1)=\tau(i)$, its inverse is given by $S \rightarrow \pi_{S}$. Thus, in order to complete the proof of Theorem 1.2 it only remains to show that

$$
\mathrm{c}(a ; R(\pi))=\prod_{i=1}^{n}\left[\begin{array}{l}
\sigma_{i}-1 \\
a_{i}-1
\end{array}\right] \frac{1-q^{\sigma_{i}}}{1-q^{\pi\left(\sigma_{i}\right)}} .
$$

By (1.8) this is equivalent to showing that

$$
\mathrm{c}(a ; R(\pi))=\left[\begin{array}{c}
|a| \\
a
\end{array}\right] \prod_{i=1}^{n} \frac{1-q^{a_{i}}}{1-q^{a_{\pi(1)}+\cdots+a_{\pi(i)}}} .
$$

According to Lemma 3.1 .

$$
\mathrm{c}(a ; R(\pi))=\frac{F_{R(\pi)}\left(c_{1}, c_{2}, \ldots, c_{n}\right)}{\phi_{1}^{\prime}\left(c_{1}\right) \phi_{2}^{\prime}\left(c_{2}\right) \cdots \phi_{n}^{\prime}\left(c_{n}\right)},
$$

where $\phi_{i}(z)=\prod_{a \in A_{i}}(z-a)$ and $c_{i}=q^{\alpha_{i}}$, with $\alpha_{\pi(i)}=a_{\pi(1)}+\cdots+a_{\pi(i-1)}$. Define $s_{1}, \ldots, s_{n+1}$ by

$$
\begin{gathered}
s_{i}:=\alpha_{\pi(i)}=a_{\pi(1)}+\cdots+a_{\pi(i-1)}=|a|-a_{\pi(i)}-a_{\pi(i+1)}-\cdots-a_{\pi(n)}, \\
s=s_{n+1}:=a_{\pi(1)}+\cdots+a_{\pi(n)}=|a|
\end{gathered}
$$


so that $s_{i+1}=s_{i}+a_{\pi(i)}$. In view of the definitions of $A_{i}, B_{\tau(i)}$ and the relation $\pi(i)=\tau(n-i+1)$ we have

$$
\phi_{\pi(i)}(z)=\frac{\prod_{l=0}^{|a|-a_{\pi(i)}}\left(z-q^{l}\right)}{\prod_{j=i+2}^{n+1}\left(z-q^{|a|-a_{\pi(i)}-\left(a_{\pi(j)}+a_{\pi(j+1)}+\cdots+a_{\pi(n)}\right)}\right)} .
$$

Therefore

$$
\begin{aligned}
\phi_{\pi(i)}^{\prime}\left(q^{\alpha_{\pi(i)}}\right)= & \frac{\prod_{l=0}^{\alpha_{\pi(i)}-1}\left(q^{\alpha_{\pi(i)}}-q^{l}\right) \cdot \prod_{l=\alpha_{\pi(i)}+1}^{s-a_{\pi(i)}}\left(q^{\alpha_{\pi(i)}}-q^{l}\right)}{\prod_{j=i+2}^{n+1}\left(q^{\alpha_{\pi(i)}}-q^{s-a_{\pi(i)}-\left(a_{\pi(j)}+a_{\pi(j+1)}+\cdots+a_{\pi(n)}\right)}\right)} \\
= & \frac{\prod_{l=0}^{n} q^{l}\left(q^{s_{i}-l}-1\right) \cdot \prod_{l=s_{i}+1}^{s-a_{\pi(i)}} q^{s_{i}}\left(1-q^{l-s_{i}}\right)}{\prod_{j=i+1}^{n} q^{\alpha_{\pi(i)}}\left(1-q^{\left.a_{\pi(i+1)}+a_{\pi(i+2)}+\cdots+a_{\pi(j)}\right)}\right)} \\
= & \frac{(-1)^{s_{i}} q^{t_{i}}(q)_{s_{i}}(q)_{s-s_{i+1}}}{\prod_{j=i+1}^{n}\left(1-q^{s_{j+1}-s_{i+1}}\right)}
\end{aligned}
$$

with $t_{i}=\left(\begin{array}{c}s_{i} \\ 2\end{array}\right)+s_{i}\left(s-s_{i+1}\right)-(n-i) s_{i}$. Next we split $F_{R(\pi)}\left(q^{\alpha_{1}}, q^{\alpha_{2}}, \ldots, q^{\alpha_{n}}\right)$ into the product $(-1)^{|R(\pi)|} \Pi_{<} \Pi_{>}$, where

$$
\begin{aligned}
\Pi_{<} & :=\prod_{\substack{\pi(u)<\pi(v) \\
u<v}}\left(\prod_{k=0}^{a_{\pi(u)}-1}\left(q^{\alpha_{\pi(v)}}-q^{\alpha_{\pi(u)}+k}\right) \cdot \prod_{k=1}^{a_{\pi(v)}-1}\left(q^{\alpha_{\pi(u)}}-q^{\alpha_{\pi(v)}+k}\right)\right) \\
& =\prod_{\substack{\pi(u)<\pi(v) \\
u<v}}\left(\prod_{k=0}^{a_{\pi(u)}-1} q^{s_{u}+k}\left(q^{s_{v}-s_{u}-k}-1\right) \cdot \prod_{k=1}^{a_{\pi(v)}-1} q^{s_{u}}\left(1-q^{s_{v}-s_{u}+k}\right)\right)
\end{aligned}
$$

and

$$
\begin{aligned}
\Pi_{>} & =\prod_{\substack{\pi(u)<\pi(v) \\
u>v}}\left(\prod_{k=0}^{a_{\pi(u)}-1}\left(q^{\alpha_{\pi(v)}}-q^{\alpha_{\pi(u)}+k}\right) \cdot \prod_{k=1}^{a_{\pi(v)}-1}\left(q^{\alpha_{\pi(u)}}-q^{\alpha_{\pi(v)}+k}\right)\right) \\
& =\prod_{\substack{\pi(u)<\pi(v) \\
u>v}}\left(\prod_{k=0}^{a_{\pi(u)}-1} q^{s_{v}}\left(1-q^{s_{u}-s_{v}+k}\right) \cdot \prod_{k=1}^{a_{\pi(v)}-1} q^{s_{v}+k}\left(q^{s_{u}-s_{v}-k}-1\right)\right) .
\end{aligned}
$$


Thus,

$$
\begin{array}{r}
\Pi_{<}=(-1)^{s_{<}} q^{t<} \prod_{\substack{i<j \\
\pi(i)<\pi(j)}}\left(\frac{(q)_{s_{j}-s_{i}}}{(q)_{s_{j}-s_{i+1}}} \cdot \frac{(q)_{s_{j+1}-s_{i}-1}}{(q)_{s_{j}-s_{i}}}\right) \\
=(-1)^{s_{<}} q^{t<} \prod_{\substack{i<j \\
\pi(i)<\pi(j)}}\left(\frac{1}{1-q^{s_{j+1}-s_{i}}} \cdot \frac{(q)_{s_{j+1}-s_{i}}}{(q)_{s_{j}-s_{i+1}}}\right)
\end{array}
$$

holds with

$$
s_{<}=\sum_{\substack{i<j \\
\pi(i)<\pi(j)}} a_{\pi(i)}, \quad t_{<}=\sum_{\substack{i<j \\
\pi(i)<\pi(j)}}\left(\left(\begin{array}{c}
a_{\pi(i)} \\
2
\end{array}\right)+\left(a_{\pi(i)}+a_{\pi(j)}-1\right) s_{i}\right) .
$$

Similarly, with the notation

$$
s_{>}=\sum_{\substack{i<j \\
\pi(i)>\pi(j)}}\left(a_{\pi(i)}-1\right), \quad t_{>}=\sum_{\substack{i<j \\
\pi(i)>\pi(j)}}\left(\left(\begin{array}{c}
a_{\pi(i)} \\
2
\end{array}\right)+\left(a_{\pi(i)}+a_{\pi(j)}-1\right) s_{i}\right)
$$

we can rewrite $\Pi_{>}$as

$$
\Pi_{>}=(-1)^{s_{>}} q^{t_{>}} \prod_{\substack{i<j \\ \pi(i)>\pi(j)}}\left(\frac{1}{1-q^{s_{j+1}-s_{i}}} \cdot \frac{(q)_{s_{j+1}-s_{i}}}{(q)_{s_{j}-s_{i+1}}}\right) .
$$

Consequently,

$$
\begin{aligned}
F_{R(\pi)}\left(q^{\alpha_{1}}, q^{\alpha_{2}}, \ldots, q^{\alpha_{n}}\right) & \\
& =(-1)^{|R(\pi)|+s_{<}+s_{>}} q^{t<+t>} \prod_{1 \leq i<j \leq n}\left(\frac{1}{1-q^{s_{j+1}-s_{i}}} \cdot \frac{(q)_{s_{j+1}-s_{i}}}{(q)_{s_{j}-s_{i+1}}}\right) \\
& =(-1)^{\sum_{i=1}^{n} s_{i}} q^{t_{<}+t_{>}} \prod_{1 \leq i<j \leq n} \frac{1}{1-q^{s_{j+1}-s_{i}}} \cdot \prod_{i=1}^{n} \frac{(q)_{s_{i}}(q)_{s-s_{i}}}{(q)_{s_{i+1}-s_{i}}}
\end{aligned}
$$

where we have used that $s_{n+1}=s, s_{1}=0$ and $|R(\pi)|+s_{<}+s_{>}=\sum_{i<j} a_{\pi(i)}=$ $\sum_{j=1}^{n} s_{j}$. It thus follows that in the expression

$$
\mathrm{c}(a ; R(\pi))=\frac{F_{R(\pi)}\left(q^{\alpha_{1}}, q^{\alpha_{2}}, \ldots, q^{\alpha_{n}}\right)}{\phi_{1}^{\prime}\left(q^{\alpha_{1}}\right) \phi_{2}^{\prime}\left(q^{\alpha_{2}}\right) \cdots \phi_{n}^{\prime}\left(q^{\alpha_{n}}\right)}
$$

the powers of -1 cancel out. So do the powers of $q$, according to the following observation.

Claim 3.4. $t_{<}+t_{>}=\sum_{i=1}^{n} t_{i}$.

Proof. Using

$$
\sum_{1 \leq i<j \leq n} a_{\pi(j)} s_{i}=\sum_{i=1}^{n} s_{i} \sum_{j=i+1}^{n}\left(s_{j+1}-s_{j}\right)=\sum_{i=1}^{n} s_{i}\left(s-s_{i+1}\right),
$$

eliminating all other $a_{\pi(i)}$ by $a_{\pi(i)}=s_{i+1}-s_{i}$ and finally using $\left(\begin{array}{c}a-b \\ 2\end{array}\right)=\left(\begin{array}{l}a \\ 2\end{array}\right)+\left(\begin{array}{l}b \\ 2\end{array}\right)+$ $(1-a) b$, we find

$$
t_{<}+t_{>}=\sum_{i=1}^{n}\left[(n-i)\left(\left(\begin{array}{c}
s_{i+1} \\
2
\end{array}\right)-\left(\begin{array}{c}
s_{i} \\
2
\end{array}\right)\right)-(n-i) s_{i}+s_{i}\left(s-s_{i+1}\right)\right] .
$$


Recalling that $s_{1}=0$, the sum over the binomial coefficients telescopes to $\sum_{i}\left(\begin{array}{c}s_{i} \\ 2\end{array}\right)$, establishing the claim.

Finally,

$$
\begin{aligned}
c(a ; R(\pi)) & =\frac{\prod_{1 \leq i<j \leq n} \frac{1}{1-q^{s_{j+1}-s_{i}}} \cdot \prod_{i=1}^{n} \frac{(q)_{s_{i}}(q)_{s-s_{i}}}{(q)_{s_{i+1}-s_{i}}}}{\prod_{i=1}^{n} \frac{(q)_{s_{i}}(q)_{s-s_{i+1}}}{\prod_{j=i+1}^{n}\left(1-q^{s_{j+1}-s_{i+1}}\right)}} \\
& =\prod_{1 \leq i<j \leq n} \frac{1-q^{s_{j+1}-s_{i+1}}}{1-q^{s_{j+1}-s_{i}}} \cdot \prod_{i=1}^{n} \frac{(q)_{s-s_{i}}}{(q)_{s-s_{i+1}}(q)_{s_{i+1}-s_{i}}} \\
& =\prod_{i=1}^{n} \frac{1-q^{s_{i+1}-s_{i}}}{1-q^{s_{i+1}}} \cdot \frac{(q)_{s}}{\prod_{i=1}^{n}(q)_{a_{\pi(i)}}} \\
& =\left[\begin{array}{c}
|a| \\
a
\end{array}\right] \prod_{i=1}^{n} \frac{1-q^{a_{i}}}{1-q^{a_{\pi(1)}+\cdots+a_{\pi(i)}}},
\end{aligned}
$$

as required.

3.2. Tournaments. Recall from the introduction that $I(w)$ denotes the inversion set of a permutation $w$ and $R(w)=I\left(w^{-1}\right)$. In particular, $R(w)$ contains all pairs of integers in the permutation $w$ that are not in natural order. For example, if $w=(3,4,6,1,5,7,2)$, then

$$
\begin{aligned}
I(w) & =\{(1,4),(1,7),(2,4),(2,7),(3,4),(3,5),(3,7),(5,7),(6,7)\}, \\
R(w) & =\{(1,3),(1,4),(1,6),(2,3),(2,4),(2,5),(2,6),(2,7),(5,6)\} .
\end{aligned}
$$

Now let $T$ be a tournament on $n$ vertices [4,34. That is, $T$ is a directed complete graph on $n$ labelled vertices $1, \ldots, n$ (thought of as players). The interpretation of a directed edge from vertex $i$ to vertex $j$ (also written as $i \rightarrow j$ ) is that (player) $i$ beats (player) $j$. If $i \rightarrow j$ we also write $(i, j) \in T$. A tournament is transitive if $i \rightarrow j$ and $j \rightarrow k$ implies that $i \rightarrow k$. The winner permutation $w_{\text {win }}$ of a transitive tournament is a ranking of the vertices (players) from best to worst. The set $R\left(w_{\text {win }}\right)$ precisely contains those edges of $T$ which have been reversed compared to the edges of the tournament $1 \rightarrow 2 \rightarrow \cdots \rightarrow n$.

To reformulate Theorem 1.2 in terms of tournaments we repeat the expansion (2.1). That is, we use

$$
\prod_{1 \leq i<j \leq n}\left(1-t_{i j} x_{j} / x_{i}\right)=\sum_{S \subseteq\left(\begin{array}{c}
{[n]} \\
2
\end{array}\right)}(-1)^{|S|} t_{S} \prod_{(i, j) \in S} x_{j} / x_{i},
$$

with $t_{S}=\prod_{(i, j) \in S} t_{i j}$. Then equating coefficients of $t_{S}$ in (1.9) and using that

$$
x_{j} / x_{i}\left(x_{i} / x_{j}\right)_{a_{i}}\left(q x_{j} / x_{i}\right)_{a_{j}-1}=-\left(x_{j} / x_{i}\right)_{a_{j}}\left(q x_{i} / x_{j}\right)_{a_{i}-1}
$$


yields, for any $S \subseteq\left(\begin{array}{c}{[n]} \\ 2\end{array}\right)$,

$$
\begin{gathered}
\mathrm{CT}\left[\prod_{(i, j) \in S}\left(x_{j} / x_{i}\right)_{a_{j}}\left(q x_{i} / x_{j}\right)_{a_{i}-1} \prod_{(i, j) \in \bar{S}}\left(x_{i} / x_{j}\right)_{a_{i}}\left(q x_{j} / x_{i}\right)_{a_{j}-1}\right] \\
= \begin{cases}{\left[\begin{array}{c}
|a| \\
a
\end{array}\right] \prod_{i=1}^{n} \frac{1-q^{a_{i}}}{1-q^{w\left(\sigma_{i}\right)}}} & \text { if } S=R(w) \text { for some } w \in \mathfrak{S}_{n}, \\
0 & \text { otherwise, }\end{cases}
\end{gathered}
$$

where $\bar{S}=\left(\begin{array}{c}{[n]} \\ 2\end{array}\right) \backslash S$. If we define the tournament $T$ as $T=\bar{S} \cup\{(j, i):(i, j) \in S\}$, then the left-hand side can be written as

$$
\mathrm{CT}\left[\prod_{(i, j) \in T}\left(x_{i} / x_{j}\right)_{a_{i}}\left(q x_{j} / x_{i}\right)_{a_{j}-1}\right] .
$$

To summarise, Theorem 1.2 is equivalent to the following statement about tournaments.

Theorem 3.5. Let $T$ be a tournament and $a=\left(a_{1}, \ldots, a_{n}\right)$ a sequence of positive integers. Then

$$
\begin{aligned}
\mathrm{CT}\left[\prod_{(i, j) \in T}\left(x_{i} / x_{j}\right)_{a_{i}}\left(q x_{j} / x_{i}\right)_{a_{j}-1}\right] \\
= \begin{cases}{\left[\begin{array}{c}
|a| \\
a
\end{array}\right] \prod_{i=1}^{n} \frac{1-q^{a_{i}}}{1-q^{w_{\mathrm{win}}\left(\sigma_{i}\right)}}} & \text { if } T \text { is transitive, } \\
0 & \text { if } T \text { is nontransitive. } .\end{cases}
\end{aligned}
$$

The 'nonzero part' of this theorem is [6. Theorem 2.2] and the 'zero part' is [6. Theorem 1.3], which is Bressoud and Goulden's 'Master Theorem'.

\section{KADELL'S CONJECTURE}

In this section we prove Theorem 1.3 and derive a number of generalisations involving Schur functions.

4.1. A scalar product. To make our proof of (1.13a) and 1.13b reasonably selfcontained, we begin by reviewing some basic facts about two families of polynomials known as type A Demazure characters or key polynomials; see e.g., [12, 14, 19, 26].

Throughout this section we assume that $x=\left(x_{1}, \ldots, x_{n}\right)$. For $1 \leq i \leq n-1$ let $\pi_{i}$ and $\hat{\pi}_{i}=\pi_{i}$ - id be the isobaric divided difference operators

$$
\left(\pi_{i} f\right)(x)=\frac{x_{i} f(x)-x_{i+1} f\left(\ldots, x_{i+1}, x_{i}, \ldots\right)}{x_{i}-x_{i+1}}
$$

and

$$
\left(\hat{\pi}_{i} f\right)(x)=\frac{x_{i+1} f(x)-x_{i+1} f\left(\ldots, x_{i+1}, x_{i}, \ldots\right)}{x_{i}-x_{i+1}} .
$$

Recall that $\mathscr{C}_{n}$ denotes the set of compositions of the form $v=\left(v_{1}, \ldots, v_{n}\right)$. If $v$ is a partition, i.e., $v=v^{+}$, we say that $v$ is dominant. We denote by $\bar{v}$ the composition $\bar{v}=\left(v_{n}, \ldots, v_{1}\right)$. If $\bar{v}=v^{+}$we say that $v$ is antidominant. For example, if $v=(1,0,4,1,0,3)$, then $v^{+}=(4,3,1,1)$ and $\bar{v}=(3,0,1,4,0,1)$. 
Let $s_{i} v=\left(\ldots, v_{i+1}, v_{i}, \ldots\right)$. Then the key polynomials $K_{v}(x)$ and $\hat{K}_{v}(x)$ for $v \in \mathscr{C}_{n}$ are defined by the recursion

$$
K_{s_{i} v}=\pi_{i} K_{v}, \quad \hat{K}_{s_{i} v}=\hat{\pi}_{i} \hat{K}_{v},
$$

subject to the initial conditions

$$
K_{v}(x)=\hat{K}_{v}(x)=x^{v} \quad \text { if } v \text { is dominant. }
$$

This definition is consistent since both types of isobaric divided difference operators satisfy the braid relations. (In fact, both $\left\{-\pi_{i}\right\}_{1<i<n}$ and $\left\{\hat{\pi}_{i}\right\}_{1<i<n}$ generate the 0-Hecke algebra of the symmetric group.) For $v$ an antidominant composition $K_{v}$ corresponds to a Schur function. Specifically $K_{\bar{\lambda}}=s_{\lambda}$ for $\lambda \in \mathscr{P}_{n}$.

The significance of the key polynomials to constant term identities rests with the fact that they form adjoint bases of the ring $\mathbb{Z}\left[x_{1}, \ldots, x_{n}\right]$ with respect to the scalar product

$$
\langle f, g\rangle=\mathrm{CT}\left[f\left(x_{1}, \ldots, x_{n}\right) g\left(x_{n}^{-1}, \ldots, x_{1}^{-1}\right) \prod_{1 \leq i<j \leq n}\left(1-x_{i} / x_{j}\right)\right] .
$$

More precisely,

$$
\left\langle K_{v}, \hat{K}_{w}\right\rangle=\delta_{v, \bar{w}} .
$$

This in particular implies that

$$
\left\langle s_{\lambda}, x^{\mu}\right\rangle=\delta_{\lambda \mu}
$$

We later require the generalisation of this formula to the case of nondominant monomials:

$$
\left\langle s_{\lambda}, x^{v}\right\rangle= \begin{cases}(-1)^{l(w)} & \text { if } v+\delta=w(\lambda+\delta) \text { for some } w \in \mathfrak{S}_{n}, \\ 0 & \text { otherwise }\end{cases}
$$

where $\delta:=(n-1, \ldots, 1,0)$.

4.2. Towards Kadell. Combining simple properties of the scalar product (4.1) with Theorem 1.2 we derive a number of Kadell-like constant term identities, given in Propositions 4.24.4 below. Kadell's conjecture follows from these results in a straightforward manner.

For $x=\left(x_{1}, \ldots, x_{n}\right), a=\left(a_{1}, \ldots, a_{n}\right)$ with all $a_{i}>0$ and

$$
\boldsymbol{t}=\left\{t_{i j}\right\}_{1 \leq i<j \leq n}
$$

we define

$$
D(a ; x ; \boldsymbol{t})=\prod_{1 \leq i<j \leq n}\left(x_{i} / x_{j}\right)_{a_{i}}\left(q x_{j} / x_{i}\right)_{a_{j}-1}\left(1-t_{i j} x_{j} / x_{i}\right)
$$

and

$$
D_{v, \lambda}(a ; \boldsymbol{t})=\mathrm{CT}\left[x^{-v} s_{\lambda}\left(x^{(a)}\right) D(a ; x ; \boldsymbol{t})\right],
$$

for $v \in \mathscr{C}_{n}$ and $\lambda \in \mathscr{P}_{|a|}$. As before, $x^{(a)}$ is given by (1.11). Note that $D_{v, \lambda}(a)$ defined in (1.12) corresponds to $D_{v, \lambda}(a ; \boldsymbol{t})$ for $t_{i j}=q^{a_{j}}$. If $\lambda=v^{+}$, as will often be the case, we simply write $D_{v}(a ; \boldsymbol{t})$ :

$$
D_{v}(a ; \boldsymbol{t})=D_{v, v^{+}}(a ; \boldsymbol{t})=\mathrm{CT}\left[x^{-v} s_{v^{+}}\left(x^{(a)}\right) D(a ; x ; \boldsymbol{t})\right] .
$$


With this notation (1.9) becomes

$$
D_{0}(a ; \boldsymbol{t})=W_{a}(\boldsymbol{t}) \prod_{i=1}^{n}\left[\begin{array}{l}
\sigma_{i}-1 \\
a_{i}-1
\end{array}\right],
$$

where 0 denotes the composition $(0, \ldots, 0)$. Since $D(a ; x ; \boldsymbol{t})$ is homogeneous of degree zero, $D_{v, \lambda}(a ; \boldsymbol{t})=0$ if $|\lambda| \neq|v|$.

For $w \in \mathfrak{S}_{n}$ and $b$ an arbitrary sequence of length $n$, let $w(b)=\left(b_{w(1)}, \ldots, b_{w(n)}\right)$. Also define $\left.w(\boldsymbol{t})=\left\{t_{w(i), w(j)}\right\}_{1 \leq i<j \leq n}\right)$ with $t_{i j}:=1 / t_{j i}$ for $i>j$. For example, if $n=3$ and $w=(2,3,1)$, then

$$
\begin{aligned}
D(w(a) ; w(x) & ; w(\boldsymbol{t}))=\left(1-t_{23} x_{2} / x_{3}\right)\left(1-t_{12}^{-1} x_{2} / x_{1}\right)\left(1-t_{13}^{-1} x_{3} / x_{1}\right) \\
& \times\left(x_{2} / x_{3}, x_{2} / x_{1}\right)_{a_{2}}\left(q x_{3} / x_{2}\right)_{a_{3}-1}\left(x_{3} / x_{1}\right)_{a_{3}}\left(q x_{1} / x_{2}, q x_{1} / x_{3}\right)_{a_{1}-1} .
\end{aligned}
$$

Lemma 4.1. For $w \in \mathfrak{S}_{n}$

$$
D_{v, \lambda}(a ; \boldsymbol{t})=t_{R(w)} D_{w(v), \lambda}(w(a) ; w(\boldsymbol{t}))
$$

or, when $\lambda=v^{+}$,

$$
D_{v}(a ; \boldsymbol{t})=t_{R(w)} D_{w(v)}(w(a) ; w(\boldsymbol{t})) .
$$

Proof. It is not hard to verify that

$$
D(w(a) ; w(x) ; w(\boldsymbol{t}))=\frac{D(a ; x ; \boldsymbol{t})}{t_{R(w)}} .
$$

Since $s_{\lambda}\left(w(x)^{w(a)}\right)=s_{\lambda}\left(x^{(a)}\right)$ (recall that $s_{\lambda}$ is a symmetric function) and $w(x)^{-w(v)}$ $=x^{-v}$, the result follows.

We now introduce a second set of variables $y=\left(y_{1}, \ldots, y_{m}\right)$ and write $x, y$ for the concatenation of $x$ and $y: x, y=\left(x_{1}, \ldots, x_{n}, y_{1}, \ldots, y_{m}\right)$. We also define $\boldsymbol{\tau}$ as

$$
\boldsymbol{\tau}=\left.\left\{t_{i j}\right\}_{1 \leq i<j \leq m+n}\right|_{t_{i j}=0} \text { for } i>n .
$$

If we finally denote the sequence $(a_{1}, \ldots, a_{n}, \underbrace{1, \ldots, 1}_{m \text { times }})$ by $a 1^{m}$, then an elementary calculation shows that

$$
D\left(a 1^{m} ; x, y ; \boldsymbol{\tau}\right)=D(a ; x ; \boldsymbol{t}) \prod_{1 \leq i<j \leq m}\left(1-y_{i} / y_{j}\right) \prod_{i=1}^{n} \prod_{j=1}^{m}\left(1-s_{i j} y_{j} / x_{i}\right)\left(x_{i} / y_{j}\right)_{a_{i}},
$$

where $s_{i j}:=t_{i, j+n}$. Given a partition $\lambda$, let $\lambda^{\prime}$ be its conjugate. Using the dual Cauchy identity for Schur functions [31] in the form

$$
\sum_{\lambda}(-1)^{|\lambda|} s_{\lambda}\left(u_{1}, \ldots, u_{n}\right) s_{\lambda^{\prime}}\left(v_{1}, \ldots, v_{m}\right)=\prod_{i=1}^{n} \prod_{j=1}^{m}\left(1-u_{i} v_{j}\right),
$$

we can expand

$$
\begin{aligned}
\prod_{i=1}^{n} \prod_{j=1}^{m}\left(x_{i} / y_{j}\right)_{a_{i}} & =\prod_{i=1}^{n} \prod_{j=1}^{m} \prod_{k=0}^{a_{i}-1}\left(1-q^{k} x_{i} / y_{j}\right) \\
& =\prod_{i=1}^{|a|} \prod_{j=1}^{m}\left(1-x_{i}^{(a)} / y_{j}\right)=\sum_{\lambda}(-1)^{|\lambda|} s_{\lambda}\left(x^{(a)}\right) s_{\lambda^{\prime}}\left(y^{-1}\right)
\end{aligned}
$$


where $y^{-1}:=\left(y_{1}^{-1}, \ldots, y_{m}^{-1}\right)$. Hence

$$
\begin{aligned}
& D\left(a 1^{m} ; x, y ; \boldsymbol{\tau}\right)=D(a ; x ; \boldsymbol{t}) \\
& \quad \times \sum_{\lambda}(-1)^{|\lambda|} s_{\lambda}\left(x^{(a)}\right) s_{\lambda^{\prime}}\left(y^{-1}\right) \prod_{1 \leq i<j \leq m}\left(1-y_{i} / y_{j}\right) \prod_{i=1}^{n} \prod_{j=1}^{m}\left(1-s_{i j} y_{j} / x_{i}\right) .
\end{aligned}
$$

Our next step is to take the constant term with respect to $y$, denoted by $\mathrm{CT}_{y}$. Recalling the scalar product (4.1), this gives

$$
\begin{aligned}
& \mathrm{CT}_{y}\left[D\left(a 1^{m} ; x, y ; \boldsymbol{\tau}\right)\right] \\
& \quad=D(a ; x ; \boldsymbol{t}) \sum_{\lambda}(-1)^{|\lambda|} s_{\lambda}\left(x^{(a)}\right)\left\langle\prod_{i=1}^{n} \prod_{j=1}^{m}\left(1-s_{i j} y_{j} / x_{i}\right), s_{\lambda^{\prime}}(y)\right\rangle_{y},
\end{aligned}
$$

where we have written $\langle\cdot, \cdot\rangle_{y}$ to indicate that the scalar product is with respect to $y$ instead of the usual $x$-variables.

Let $\mathscr{M}_{n, m}$ be the set of $(0,1)$-matrices of size $n \times m$ and $\mathscr{C}_{n, m}=\left\{\left(v_{1}, \ldots, v_{n}\right) \in\right.$ $\mathbb{Z}^{n}: 0 \leq v_{i} \leq m$ for all $\left.1 \leq i \leq n\right\}$ the subset of the set of compositions $\mathscr{C}_{n}$ that fit in a rectangle of size $n \times m$. For $\kappa \in \mathscr{M}_{n, m}$ denote by $r(\kappa) \in \mathscr{C}_{n, m}$ and $c(\kappa) \in \mathscr{C}_{m, n}$ the compositions encoding the row and column sums of $\kappa$ :

$$
r_{i}(\kappa)=\sum_{j=1}^{m} \kappa_{i j} \quad \text { and } \quad c_{j}(\kappa)=\sum_{i=1}^{n} \kappa_{i j} .
$$

We shall write $|\kappa|=\sum_{i j} \kappa_{i j}=|r(\kappa)|=|c(\kappa)|$ for the sum of the entries of $\kappa$. For example, if

$$
\kappa=\left(\begin{array}{lllll}
0 & 1 & 0 & 0 & 1 \\
1 & 1 & 0 & 0 & 0 \\
1 & 1 & 0 & 1 & 1 \\
0 & 0 & 0 & 1 & 0
\end{array}\right) \in \mathscr{M}_{4,5}
$$

then $r(\kappa)=(2,2,4,1), c(\kappa)=(2,3,0,2,2)$ and $|\kappa|=9$.

Let

$$
\boldsymbol{s}:=\left\{s_{i j}\right\}_{1 \leq i \leq n ; 1 \leq j \leq m}=\left\{t_{i, j+n}\right\}_{1 \leq i \leq n} ; 1 \leq j \leq m,
$$

and for $f(s)$ a polynomial in $s$ denote by $\left[\boldsymbol{s}^{\kappa}\right] f(\boldsymbol{s})$ the coefficient of the monomial $\boldsymbol{s}^{\kappa}:=\prod_{i=1}^{n} \prod_{j=1}^{m} s_{i j}^{\kappa_{i j}}$ in $f$. Since

$$
\prod_{i=1}^{n} \prod_{j=1}^{m}\left(1-s_{i j} y_{j} / x_{i}\right)=\sum_{\kappa \in \mathscr{M}_{n, m}}(-1)^{|\kappa|} y^{c(\kappa)} x^{-r(\kappa)} s^{\kappa},
$$

we have

$$
\begin{aligned}
{\left[\boldsymbol{s}^{\kappa}\right] \mathrm{CT}_{y}\left[D\left(a 1^{m} ; x, y ; \boldsymbol{\tau}\right)\right] } & \\
& =D(a ; x ; \boldsymbol{t}) \sum_{\lambda}(-1)^{|\lambda|+|\kappa|} x^{-r(\kappa)} s_{\lambda}\left(x^{(a)}\right)\left\langle y^{c(\kappa)}, s_{\lambda^{\prime}}(y)\right\rangle_{y} .
\end{aligned}
$$

Recalling (4.2), the summand on the right vanishes unless $\lambda$ is the (unique) partition such that its conjugate satisfies $\lambda^{\prime}=w\left(c(\kappa)+\delta_{m}\right)-\delta_{m}$, where $\delta_{m}:=(m-1, \ldots, 1,0)$ and $w \in \mathfrak{S}_{m}$. This in particular implies that $|\lambda|=|c(\kappa)|=|\kappa|$. Assuming such $\lambda$ and $w$, and interchanging the left- and right-hand sides, we obtain

$$
x^{-r(\kappa)} s_{\lambda}\left(x^{(a)}\right) D(a ; x ; \boldsymbol{t})=(-1)^{l(w)}\left[\boldsymbol{s}^{\kappa}\right] \mathrm{CT}_{y}\left[D\left(a 1^{m} ; x, y ; \boldsymbol{\tau}\right)\right] .
$$


As a final step we take the constant term with respect to $x$ to arrive at the following result.

Proposition 4.2. Let $a=\left(a_{1}, \ldots, a_{n}\right)$ be a sequence of positive integers, $\delta_{m}=$ $(m-1, \ldots, 1,0)$, and let $\boldsymbol{t}$ and $\boldsymbol{\tau}$ be given by (4.3) and (4.4) respectively. For $\kappa$ a $(0,1)$-matrix in $\mathscr{M}_{n, m}, \lambda \in \mathscr{C}_{|a|, m}$ a partition of $|\kappa|$ and $w \in \mathfrak{S}_{m}$ such that

$$
\lambda^{\prime}=w\left(c(\kappa)+\delta_{m}\right)-\delta_{m},
$$

we have

$$
D_{r(\kappa), \lambda}(a ; \boldsymbol{t})=(-1)^{l(w)}\left[\boldsymbol{s}^{\kappa}\right] D_{0}\left(a 1^{m} ; \boldsymbol{\tau}\right) .
$$

We should remark that one cannot choose $\kappa \in \mathscr{M}_{n, m}$ such that all conceivable row and column sums $r(\kappa) \in \mathscr{C}_{n, m}$ and $c(\kappa) \in \mathscr{C}_{m, n}$ arise. Apart from the obvious restriction $|r(\kappa)|=|c(\kappa)|$, we are bound by the Gale-Ryser theorem [7]. This theorem for example says that a pair of partitions $\mu$ and $\nu$ can arise as the row and column sums of a $(0,1)$-matrix if and only if $\mu$ is dominated by $\nu^{\prime}$. The smallest example of a constant term that does not occur in the above is $n=2, \lambda=(1,1)$ and $r(\kappa)=(2,0)$ or $(0,2)$. Whatever choice of $m \geq 2$ we make, $\max \left\{\lambda^{\prime}+\delta_{m}\right\}=m+1$ whereas $\max \left\{c(\kappa)+\delta_{m}\right\} \leq m$. Hence there is no $w \in \mathfrak{S}_{m}$ such that (4.5) holds. Despite these caveats, the above proposition is very useful since for each admissible pair $\lambda, r(\kappa)$ the constant term on the right-hand side has already been evaluated by Theorem 1.2 with $n \mapsto m+n$. In particular,

$$
D_{0}\left(a 1^{m} ; \boldsymbol{\tau}\right)=\left.\sum_{w \in \mathfrak{S}_{m+n}} c_{w}(a) t_{R(w)}\right|_{t_{i j}=0 \text { for } i>n} .
$$

The fact that $t_{i j}=0$ for $i>n$ means that only the $(m+n) ! / m$ ! permutations of $(1, \ldots, n+m)$ contribute for which each pair of integers in $n+1, \ldots, n+m$ occurs in natural order. But this in turn means that if $t_{i, j+n}=s_{i j}$ is in $t_{R(w)}$, then so must be $s_{i 1}, s_{i 2}, \ldots, s_{i, j-1}$; if $i<n$ overtakes $j>n$ but $n+1, \ldots, n+m$ are in natural order, then $i$ must also have overtaken $n+1, \ldots, j-1$. In terms of the $(0,1)$-matrices this means that each row must be a sequence of ones (possibly of zero length) followed by a sequence of zeros (possibly of zero length), otherwise the coefficient of $\boldsymbol{s}^{\kappa}$ is necessarily zero. We summarise this in our next proposition.

Proposition 4.3. Assume the conditions of Proposition 4.2. Then

$$
D_{r(\kappa), \lambda}(a ; \boldsymbol{t})=0
$$

if $\kappa$ is not a $(0,1)$-matrix such that in each row all ones precede all zeros.

A $(0,1)$-matrix $\kappa$ such that the ones in each row precede the zeros is uniquely determined by its row sums, and in particular $c_{j}(\kappa)=\left|\left\{r_{i}(\kappa): i \geq j\right\}\right|$ or, more succinctly, $c(\kappa)=\left(r^{+}(\kappa)\right)^{\prime}=: \nu^{\prime}$. For example, for

$$
\kappa=\left(\begin{array}{lllll}
1 & 1 & 0 & 0 & 0 \\
0 & 0 & 0 & 0 & 0 \\
1 & 1 & 1 & 0 & 0 \\
1 & 1 & 0 & 0 & 0
\end{array}\right) \in \mathscr{M}_{4,5}
$$

$r(\kappa)=(2,0,3,2), r^{+}(\kappa)=(3,2,2,0)$ and $c(\kappa)=(3,3,1,0,0)=\left(r^{+}(\kappa)\right)^{\prime}$. If $c(\kappa)=$ $\nu^{\prime}$, then (4.5) is solved by $(\lambda, w)=(\nu$, id $)$, leading to our next result. 
Proposition 4.4. Let $a=\left(a_{1}, \ldots, a_{n}\right)$ be a sequence of positive integers and let $\boldsymbol{t}$ and $\boldsymbol{\tau}$ be given by (4.3) and (4.4) respectively. Then, for $v \in \mathscr{C}_{n, m}$,

$$
D_{v}(a ; \boldsymbol{t})=D_{v, v^{+}}(a ; \boldsymbol{t})=\left[\prod_{i=1}^{n} \prod_{j=1}^{v_{i}} s_{i j}\right] D_{0}\left(a 1^{m} ; \boldsymbol{\tau}\right) .
$$

4.3. Proof of Kadell's conjecture. Because our results of Section 4.2 assume that all integers of the sequence $a=\left(a_{1}, \ldots, a_{n}\right)$ are positive (it is in fact easy to show that all results are true provided at most one of the $a_{i}$ is zero), we need to separately treat the case when some of the $a_{i}$ are zero.

Proof of (1.13a) for positive $a_{i}$. Let $v \in \mathscr{C}_{n, m}$ be a composition of $m$ such that $\max \left\{v_{i}\right\}<m$. Now choose $\kappa$ to be a $(0,1)$-matrix in $\mathscr{M}_{n, m}$ such that

$$
r(\kappa)=\left(v_{1}, \ldots, v_{n}\right)=v \quad \text { and } \quad c(\kappa)=\left(1^{m}\right) .
$$

In other words, each column of $\kappa$ contains a single one and the row sums of $\kappa$ (none of which has more than $m-1$ ones) form the composition $v$. Obviously, there must be a row of $\kappa$ which contains a 0 followed by a 1 , since not all ones occur in the same row and no column has more than a single one. We also note that the Gale-Ryser theorem does not cause an obstruction since there are exactly $m ! /\left(v_{1} ! \cdots v_{n} !\right)>0$ $(0,1)$-matrices such that (4.7) holds. Finally, since $c(\kappa)=\left(1^{m}\right)$, (4.5) is solved by $(\lambda, w)=((m)$, id $)$. According to Proposition 4.3 we thus have

$$
D_{v,(m)}(a ; \boldsymbol{t})=0 .
$$

Equation (1.13a) corresponds to the special case $t_{i j}=q^{a_{j}}$.

Proof of (1.13b) for positive $a_{i}$. Define

$$
W_{a}^{(k)}(\boldsymbol{t})=\sum_{\substack{w \in \mathfrak{S}_{n} \\ w(n)=k}} t_{R(w)} \prod_{i=1}^{n} \frac{1-q^{\sigma_{i}}}{1-q^{w\left(\sigma_{i}\right)}} .
$$

Instead of (1.13b) we will derive the more general identity

$$
D_{v}(a ; \boldsymbol{t})=D_{v,(m)}(a ; \boldsymbol{t})=W_{a}^{(k)}(\boldsymbol{t}) \frac{\left(q^{|a|}\right)_{m}}{\left(q^{|a|-a_{k}+1}\right)_{m}} \prod_{i=1}^{n}\left[\begin{array}{c}
\sigma_{i}-1 \\
a_{i}-1
\end{array}\right]
$$

for $v=\left(0^{k-1}, m, 0^{n-k}\right)$. From the following refinement of (1.10),

$$
\sum_{\substack{w \in \mathfrak{S}_{n} \\ w(n)=k}} w\left(\prod_{i=1}^{n} \frac{1-u_{i}}{1-u_{1} \cdots u_{i}}\right) \prod_{(i, j) \in R(w)} u_{j}=\frac{u_{k+1} \cdots u_{n}\left(1-u_{k}\right)}{1-u_{1} \cdots u_{n}},
$$

it follows that

$$
\left.W_{a}^{(k)}(\boldsymbol{t})\right|_{t_{i j}=q^{a_{j}}}=q^{\sigma_{n}-\sigma_{k}} \frac{1-q^{a_{k}}}{1-q^{|a|}} \prod_{i=1}^{n} \frac{1-q^{\sigma_{i}}}{1-q^{a_{i}}},
$$

resulting in (1.13b).

It remains to show (4.8) and (4.9). Starting with the latter, we will simultaneously prove (1.10) (with $m \mapsto n$ ) and (4.9). If we denote the sum sides of these identities by $g(u)$ and $g_{k}(u)$, then

$$
g(u)=\sum_{k=1}^{n} g_{k}(u) \quad \text { and, for } k \geq 1, \quad g_{k}(u)=\frac{u_{k+1} \cdots u_{n}\left(1-u_{k}\right)}{1-u_{1} \cdots u_{n}} g\left(u^{(k)}\right),
$$


where $u^{(k)}=\left(u_{1}, \ldots, u_{k-1}, u_{k+1}, \ldots, u_{n}\right)$. These two equations imply a recurrence for $g(u)$. Thanks to the initial condition $g(-)=1$ and some telescoping, this is trivially solved by $g(u)=1$, establishing both (1.10) and (4.9).

To prove (4.8) we let $v=(0, \ldots, 0, m)$ in (4.6). Then

$$
D_{(0, \ldots, 0, m)}(a, \boldsymbol{t})=\left[s_{n 1} \ldots s_{n m}\right] D_{0}\left(a 1^{m} ; \boldsymbol{\tau}\right) .
$$

We now essentially repeat the reasoning that led to Proposition 4.3. The fact that $t_{i j}=0$ for $i>n$ means that only the $(m+n) ! / m$ ! permutations of $(1, \ldots, n+m)$ contribute to $D_{0}\left(a 1^{m} ; \boldsymbol{\tau}\right)$ for which each pair of integers in $n+1, \ldots, n+m$ occurs in natural order. Since we further need to take the coefficient of $s_{n 1} \ldots s_{n m}$, only those permutations $w$ contribute for which $t_{R(w)}$ contains the subword $t_{n, n+1} \ldots t_{n, m}$ but none of the letters $t_{i, j+n}$ for $i<n$ and $j \geq 1$. These are exactly the $(n-1)$ ! permutations of the form $w=(\pi, n+1, \ldots, m+n, n)$, where $\pi \in \mathfrak{S}_{n-1}$. For such a permutation, and $a_{n+1}=\cdots=a_{n+m}=1$,

$$
\prod_{i=1}^{m+n} \frac{1-q^{\sigma_{i}}}{1-q^{w\left(\sigma_{i}\right)}}\left[\begin{array}{l}
\sigma_{i}-1 \\
a_{i}-1
\end{array}\right]=\frac{\left(q^{|a|}\right)_{m}}{\left(q^{|a|-a_{n}+1}\right)_{m}} \prod_{i=1}^{n-1} \frac{1-q^{\sigma_{i}}}{1-q^{\pi\left(\sigma_{i}\right)}} \prod_{i=1}^{n}\left[\begin{array}{l}
\sigma_{i}-1 \\
a_{i}-1
\end{array}\right] .
$$

Thanks to Theorem 1.2 we thus find

$$
\begin{aligned}
D_{(0, \ldots, 0, m)}(a ; \boldsymbol{t}) & =\frac{\left(q^{|a|}\right)_{m}}{\left(q^{|a|-a_{n}+1}\right)_{m}} \prod_{i=1}^{n}\left[\begin{array}{c}
\sigma_{i}-1 \\
a_{i}-1
\end{array}\right] \cdot \sum_{\pi \in \mathfrak{S}_{n-1}} t_{R(\pi)} \prod_{i=1}^{n-1} \frac{1-q^{\sigma_{i}}}{1-q^{\pi\left(\sigma_{i}\right)}} \\
& =W_{a}^{(n)}(\boldsymbol{t}) \frac{\left(q^{|a|}\right)_{m}}{\left(q^{|a|-a_{n}+1}\right)_{m}} \prod_{i=1}^{n}\left[\begin{array}{c}
\sigma_{i}-1 \\
a_{i}-1
\end{array}\right] .
\end{aligned}
$$

This settles the $k=n$ case of (4.8).

To deal with the remaining cases, let $s_{k}$ denote the $k$ th adjacent (or simple) transposition. Then, by the $w=s_{k}$ case of Lemma 4.1,

$$
D_{\left(0^{k-1}, m, 0^{n-k}\right)}(a ; \boldsymbol{t})=t_{k, k+1} D_{\left(0^{k-2}, m, 0^{n-k+1}\right)}\left(s_{k}(a) ; s_{k}(\boldsymbol{t})\right) \quad 1 \leq k \leq n-1 .
$$

All we need to do is show that the claimed right-hand side of (4.8) satisfies this same recursion. To this end let $w \in \mathfrak{S}_{n}$ such that $w(n)=k$ and let $W_{a}^{(k)}(\boldsymbol{t} ; w)$ denote the right-hand side but without the sum over $\mathfrak{S}_{n}$ :

$$
W_{a}^{(k)}(\boldsymbol{t} ; w)=\frac{\left(q^{|a|}\right)_{m}}{\left(q^{|a|-a_{k}+1}\right)_{m}} \prod_{i=1}^{n}\left[\begin{array}{c}
\sigma_{i}-1 \\
a_{i}-1
\end{array}\right] \cdot t_{R(w)} \prod_{i=1}^{n} \frac{1-q^{\sigma_{i}}}{1-q^{w\left(\sigma_{i}\right)}} .
$$

It is a straightforward exercise to verify that

$$
W_{a}^{(k)}(\boldsymbol{t} ; w)=t_{k, k+1} W_{s_{k}(a)}^{(k+1)}\left(s_{k}(\boldsymbol{t}) ; \hat{w}\right),
$$

where $\hat{w}$ is the same permutation as $w$ except for the fact that the numbers $k$ and $k+1$ have swapped position. (In particular, $\hat{w}(n)=k+1$ as it should.) Summing $w$ over $\mathfrak{S}_{n}($ such that $w(n)=k)$ does the rest.

Proof of Theorem 1.3 when not all $a_{i}>0$. We may assume that not all $a_{i}$ are zero since

$$
D_{v, \lambda}(0, \ldots, 0)=\delta_{v, 0} \delta_{\lambda, 0} .
$$

Given $a=\left(a_{1}, \ldots, a_{n}\right)$ and $I \subseteq\{1, \ldots, n\}$, let $a^{I}$ denote the sequence obtained from $a$ by deleting all $a_{i}$ for $i \in I$ and let $a_{I}$ be the sequence of the deleted $a_{i}$. For example, if $n=7$, then $a^{\{3,5,6\}}=\left(a_{1}, a_{2}, a_{4}, a_{7}\right)$ and $a_{\{3,5,6\}}=\left(a_{3}, a_{5}, a_{6}\right)$. 
Now let $I$ denote the index-set of those $a_{i}$ that are zero, so that the entries of $a^{I}$ are all strictly positive. Recalling the third of our remarks made after (1.12) we have

$$
D_{v,(m)}(a)=D_{v^{I},(m)}\left(a^{I}\right) \prod_{i \in I} \delta_{v_{i}, 0} .
$$

To prove that (1.13b) for $I \neq \emptyset$ is true there are two cases to consider. First, if $k \in I$ (i.e., $a_{k}=0$ ), then the right-hand side vanishes due to the factor $\delta_{v_{k}, 0}$ in (4.10) and the fact that $v_{k}=m>0$. But the left side also vanishes due to the factor $\left(1-q^{a_{k}}\right)$. Next, if $k \notin I$, then $v_{i}=0$ for all $i \in I$. Hence (4.10) simplifies to

$$
D_{v,(m)}(a)=D_{v^{I},(m)}\left(a^{I}\right)=\frac{q^{\sigma_{n}-\sigma_{k}}\left(1-q^{a_{k}}\right)\left(q^{|a|}\right)_{m}}{\left(1-q^{|a|}\right)\left(q^{|a|-a_{k}+1}\right)_{m}}\left[\begin{array}{c}
|a| \\
a
\end{array}\right],
$$

where the second equality follows from (1.13b) for positive $a_{i}$.

Finally, to see that (1.13a) for $I \neq \emptyset$ is true we note that $v^{+} \neq(m)$ implies that $\left(v^{I}\right)^{+} \neq(m)$. Hence, by (1.13a) for positive $a_{i}$, (4.10) yields

$$
D_{v,(m)}(a)=0 .
$$

4.4. Beyond Kadell. One can consider more general applications of Propositions 4.2 4.4 than Kadell's conjecture, and in this section we present the full details of one further example.

In Proposition 4.4 take $v=\bar{\lambda}$ with $\lambda \in \mathscr{P}_{n}$ such that $\lambda_{1}=m$. Then

$$
D_{\bar{\lambda}}(a ; \boldsymbol{t})=\left[\prod_{i=1}^{n} \prod_{j=1}^{\lambda_{n-i+1}} s_{i j}\right] D_{0}\left(a 1^{m} ; \boldsymbol{\tau}\right) .
$$

As before, we need to determine which permutations in $\mathfrak{S}_{m+n}$ contribute to the right-hand side. This can simply be read from a diagrammatic representation of the right as we will illustrate through an example.

Let $m=3, n=4$ and $\bar{\lambda}=(0,1,3,3)$, and represent the set $s$ as well as the composition $\bar{\lambda}$ by a filling of an $m \times n$ rectangle as follows:

$$
\begin{array}{lll}
t_{15} & t_{16} & t_{17} \\
t_{25} & t_{26} & t_{27} \\
t_{35} & t_{36} & t_{37} \\
t_{45} & t_{46} & t_{47}
\end{array}
$$

Taking the coefficient of $\prod_{i=1}^{n} \prod_{j=1}^{\lambda_{n-i+1}} s_{i j}$ means that we need to take the coefficient of

$$
t_{15}^{0} t_{16}^{0} t_{17}^{0} t_{25}^{1} t_{26}^{0} t_{27}^{0} t_{35}^{1} t_{36}^{1} t_{37}^{1} t_{45}^{1} t_{46}^{1} t_{47}^{1}=t_{25} t_{35} t_{36} t_{37} t_{45} t_{46} t_{47} .
$$

Which permutations in $\mathfrak{S}_{4+3}$ contribute can now be read from the diagram. The numbers $5,6,7$ need to be in natural order since $t_{56}=t_{57}=t_{67}=0$. Because $t_{45}, t_{46}$ and $t_{47}$ are covered by the diagram of $\bar{\lambda}$, it follows that 4 occurs after $5,6,7$. Because $t_{35}, t_{36}$ and $t_{37}$ are covered by $\bar{\lambda}$, it follows that 3 also occurs after $5,6,7$. Because $t_{25}$ is covered by $\bar{\lambda}$, but $t_{26}$ is not, the number 2 comes after 5 but before 6 . Because $t_{15}$ is not covered, the number 1 comes before $5,6,7$. As a result only two permutations contribute:

$$
(1,5,2,6,7,3,4) \text { and }(1,5,2,6,7,4,3) \text {. }
$$


Having worked out this example in full detail it is clear that the following somewhat simpler diagram than the above staircase encodes exactly the same information:

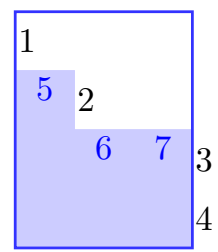

Here numbers occurring in the same column (such as 3,4) may be permuted, but numbers in the same row (such as 6,7) have their relative ordering fixed.

Given $\lambda=\left(\lambda_{1}, \ldots, \lambda_{n}\right) \in \mathscr{C}_{n}$ such that $\lambda_{1}=m$, let $\mathfrak{m}_{i}=\mathfrak{m}_{i}(\lambda)$ for $0 \leq i \leq m$ be the multiplicity of $i$ in $\lambda$ :

$$
\mathfrak{m}_{i}=\left|\left\{\lambda_{j}: \lambda_{j}=i\right\}\right| .
$$

Then the number of numbers in the $i$ th column $(0 \leq i \leq m)$ of the above-type diagram is given by $\mathfrak{m}_{i}(\lambda)$, so that the sum over $\mathfrak{S}_{m+n}$ reduces to a sum over $\mathfrak{S}_{\mathfrak{m}_{0}} \times \cdots \times \mathfrak{S}_{\mathfrak{m}_{m}}$. In the case of our example, $\mathfrak{m}_{0}=1, \mathfrak{m}_{1}=1, \mathfrak{m}_{2}=0, \mathfrak{m}_{3}=2$, resulting in a sum over $\mathfrak{S}_{2}$ instead of $\mathfrak{S}_{7}$.

We conclude by applying the above considerations to the case of strict partitions, i.e., $\lambda=\left(\lambda_{1}, \ldots, \lambda_{n}\right)$ with $\lambda_{i}>\lambda_{i+1}$ for all $1 \leq i \leq n-1$. Then all multiplicities are 1 so that there is only one remaining permutation:

$$
\begin{aligned}
\left(n+1, \ldots, n+\lambda_{n}, 1, n+\lambda_{n}+1, \ldots, n+\lambda_{n-1}, 2, \ldots,\right. & \\
n & \left.-1, n+\lambda_{2}+1, \ldots, n+\lambda_{1}, n\right) .
\end{aligned}
$$

Therefore

$$
D_{\bar{\lambda}}(a ; \boldsymbol{t})=\prod_{i=1}^{n}\left[\begin{array}{c}
\bar{\lambda}_{i}+\sigma_{i}-1 \\
a_{i}-1
\end{array}\right] .
$$

Applying Lemma 4.1 with $v=\bar{\lambda}$, this yields

$$
D_{w^{-1}(\bar{\lambda})}(a ; \boldsymbol{t})=w\left(\prod_{i=1}^{n}\left[\begin{array}{c}
\bar{\lambda}_{i}+\sigma_{i}-1 \\
a_{i}-1
\end{array}\right]\right) t_{R(w)},
$$

where

$$
w\left(\left[\begin{array}{c}
\bar{\lambda}_{i}+\sigma_{i}-1 \\
a_{i}-1
\end{array}\right]\right)=\left[\begin{array}{c}
\bar{\lambda}_{i}+a_{w(1)}+\cdots+a_{w(i)}-1 \\
a_{w(i)}-1
\end{array}\right] .
$$

Finally taking $t_{i j}=q^{a_{j}}$ results in our final proposition.

Proposition 4.5. Let $\lambda \in \mathscr{P}_{n}$ be a strict partition, i.e., $\lambda_{1}>\lambda_{2}>\cdots>\lambda_{n} \geq 0$, and set $\bar{\lambda}=\left(\lambda_{n}, \ldots, \lambda_{1}\right)$. Then, for $a_{1}, \ldots, a_{n}$ positive integers and $w \in \mathfrak{S}_{n}$,

$$
D_{w^{-1}(\bar{\lambda})}(a)=w\left(\prod_{i=1}^{n}\left[\begin{array}{c}
\bar{\lambda}_{i}+\sigma_{i}-1 \\
a_{i}-1
\end{array}\right]\right) q^{\sum_{(i, j) \in R(w)} a_{j}} .
$$

When $w=(n, \ldots, 2,1)$ is the permutation of maximal length we obtain (1.14). 


\section{An application to some $q$-Dyson coefficients}

A result concerning coefficients of the $q$-Dyson product other than the constant term is a theorem of $\mathrm{Lv}$, Xin and Zhou [28, which can be rephrased as an evaluation of $D_{v, 0}(a)$ for certain $v \in \mathbb{Z}^{n}$ (as opposed to $v \in \mathscr{C}_{n}$ ) as follows.

Theorem 5.1. Let $v \in \mathbb{Z}^{n}$ such that $|v|=0, \max \{v\} \leq 1$ and $v_{1}=1$. Let $I$ be the index-set of the positive $v_{i}$, i.e., $I=\left\{1 \leq i \leq n: v_{i}=1\right\}$. Then

$$
\mathrm{CT}\left[x^{-v} \prod_{1 \leq i<j \leq n}\left(x_{i} / x_{j}\right)_{a_{i}}\left(q x_{j} / x_{i}\right)_{a_{j}}\right]=\left[\begin{array}{c}
|a| \\
a
\end{array}\right] \sum_{J \subseteq I}(-1)^{|J|} q^{E(J)} \frac{1-q^{a_{J}}}{1-q^{1+|a|-a_{J}}},
$$

where $a_{J}:=\sum_{j \in J} a_{j}$ and

$$
E(J)=\sum_{\substack{1 \leq i \leq j \leq n \\ j \notin J}} v_{i} a_{j}
$$

The technical condition $v_{1}=1$ can be easily dropped by the application of a simple transformation which will be described later, but the formula thus obtained is less attractive. The above theorem extends the equal parameter case of Stembridge's first layer formulas for characters of $\operatorname{SL}(n, \mathbb{C})$ [35] and contains as special cases a number of earlier conjectures of Sills 32 .

In their report the anonymous referee asked the question as to whether some of these more general Dyson-type identities follow from our main theorems. In answer to this question we will now show that Kadell's orthogonality conjecture of Theorem 1.3 indeed implies one of Sills' ex-conjectures [32, Conjecture 1.2].

Theorem 5.2 (cf. [28, Corollary 1.4]). Let $a=\left(a_{1}, \ldots, a_{n}\right)$ be of sequence of nonnegative integers and $1 \leq r, s \leq n$ a pair of distinct integers. Then

$$
\mathrm{CT}\left[\left(x_{r} / x_{s}\right) \prod_{1 \leq i<j \leq n}\left(x_{i} / x_{j}\right)_{a_{i}}\left(q x_{j} / x_{i}\right)_{a_{j}}\right]=-q^{E_{r, s}} \frac{1-q^{a_{s}}}{1-q^{1+|a|-a_{s}}}\left[\begin{array}{c}
|a| \\
a
\end{array}\right],
$$

where

$$
E_{r, s}=\chi(r<s)+\sum_{i=s+1}^{r-1} a_{i}
$$

In the above the summation is understood cyclically, i.e.,

$$
\sum_{i=s+1}^{r-1} a_{i}=\sum_{i=s+1}^{n} a_{i}+\sum_{i=1}^{r-1} a_{i}
$$

when $r<s$.

In the proof we will exploit some properties of the following transformation. For a Laurent polynomial $L(x)$, define the $q$-shifted cyclic action $\gamma$ on $L$ as

$$
\gamma\left(L\left(x_{1}, x_{2}, \ldots, x_{n-1}, x_{n}\right)\right)=L\left(x_{2}, x_{3}, \ldots, x_{n}, x_{1} / q\right) .
$$

Abbreviate the $q$-Dyson product as $D(a ; x)$. Then $\mathrm{CT}[L]=\mathrm{CT}[\gamma(L)]$ and

$$
\gamma^{-1}(D(a ; x))=D(\gamma(a) ; x)
$$

where $\gamma(a):=\left(a_{2}, \ldots, a_{n}, a_{1}\right)$; cf. [28, Lemma 2.1]. 
Proof of Theorem 5.2. Fixing $1<k \leq n$ and $m=1$, 1.13b) reads

$$
\mathrm{CT}\left[\sum_{i=1}^{n} \frac{1-q^{a_{i}}}{1-q} \cdot \frac{x_{i}}{x_{k}} D(a ; x)\right]=\frac{q^{a_{k+1}+\cdots+a_{n}}\left(1-q^{a_{k}}\right)}{1-q^{|a|-a_{k}+1}}\left[\begin{array}{c}
|a| \\
a
\end{array}\right] .
$$

Applying the same formula but with $k-1$ instead of $k$ and $\gamma(a)$ instead of $a$, and with the convention $a_{n+1}=a_{1}$, we obtain

$$
\mathrm{CT}\left[\sum_{i=1}^{n} \frac{1-q^{a_{i}+1}}{1-q} \cdot \frac{x_{i}}{x_{k}} D(\gamma(a) ; x)\right]=\frac{q^{a_{k+1}+\cdots+a_{n+1}}\left(1-q^{a_{k}}\right)}{1-q^{|a|-a_{k}+1}}\left[\begin{array}{c}
|a| \\
a
\end{array}\right] .
$$

By the above-mentioned properties of $\gamma$, the left-hand side may be reinterpreted as

$$
\mathrm{CT}\left[\left(\frac{1-q^{a_{1}}}{q(1-q)} \cdot \frac{x_{1}}{x_{k}}+\sum_{i=2}^{n} \frac{1-q^{a_{i}}}{1-q} \cdot \frac{x_{i}}{x_{k}}\right) D(a ; x)\right] .
$$

Subtracting the two equations thus obtained we find that

$$
\left(1-q^{-1}\right) \mathrm{CT}\left[\frac{1-q^{a_{1}}}{1-q} \cdot \frac{x_{1}}{x_{k}} D(a ; x)\right]=\frac{\left(1-q^{a_{1}}\right) q^{a_{k+1}+\cdots+a_{n}}\left(1-q^{a_{k}}\right)}{1-q^{|a|-a_{k}+1}}\left[\begin{array}{c}
|a| \\
a
\end{array}\right] .
$$

This establishes Theorem 5.2 in the special case when $r=1$. Using the convention $x_{0}=x_{n}$, the full content of the theorem follows by a repeated application of the identity

$$
\mathrm{CT}\left[\left(x_{r} / x_{s}\right) D(a ; x)\right]=q^{\chi(r=1)-\chi(s=1)} \mathrm{CT}\left[\left(x_{r-1} / x_{s-1}\right) D(\gamma(a) ; x)\right] .
$$

As briefly outlined below, Theorem 5.2 can also be obtained directly by using the polynomial method we employed to prove Theorem 1.2. Note that we may clearly assume that $s=1$ and that all $a_{i}$, with the possible exception of $a_{r}$, are positive. Accordingly, we want to compute the coefficient of the monomial

$$
\left(x_{1} / x_{r}\right) \prod_{i=1}^{n} x_{i}^{|a|-a_{i}}
$$

in the homogeneous polynomial

$$
F(x)=\prod_{1 \leq i<j \leq n}\left(\prod_{k=0}^{a_{i}-1}\left(x_{j}-x_{i} q^{k}\right) \cdot \prod_{k=1}^{a_{j}}\left(x_{i}-x_{j} q^{k}\right)\right) .
$$

This can be done efficiently using Lemma 3.1 if the sets $A_{i}$ therein are chosen as follows. Let $A_{i}=\left\{q^{\alpha_{i}}: \alpha_{i} \in B_{i}\right\}$, where

$$
B_{1}=\left\{0,1, \ldots,|a|-a_{1}+1\right\}, \quad B_{r}=\left\{0, \ldots,|a|-a_{r}\right\} \backslash\left\{\sum_{i=2}^{r-1} a_{i}\right\}
$$

and $B_{i}=\left\{0, \ldots,|a|-a_{i}\right\}$ otherwise. The sets $A_{i}$ clearly have the right cardinalities. Now there is exactly one element $c \in A_{1} \times \cdots \times A_{n}$ such that $F(c) \neq 0$. Indeed, assume that $c_{i}=q^{\alpha_{i}} \in A_{i}$ and $F(c) \neq 0$. Then all $\alpha_{i}$ are distinct, with the possible exception of $a_{r}$ being equal to $a_{j}$ for some $j>r$. Moreover, $\alpha_{j} \geq \alpha_{i}+a_{i}+\chi(j<i)$ holds for $\alpha_{j}>\alpha_{i}$. Next consider the unique permutation $\pi \in \mathfrak{S}_{n}$ for which

$$
0 \leq \alpha_{\pi(1)} \leq \alpha_{\pi(2)} \leq \cdots \leq \alpha_{\pi(n)} \leq|a|-a_{\pi(n)}+\chi(\pi(n)=1) .
$$


Here $\pi(i)=r$ is assumed in the case of $\alpha_{\pi(i+1)}=\alpha_{\pi(i)}$. We can argue that

$$
|a|-a_{\pi(n)}=\sum_{i=1}^{n-1} a_{\pi(i)} \leq \sum_{i=1}^{n-1}\left(\alpha_{\pi(i+1)}-\alpha_{\pi(i)}\right)=\alpha_{\pi(n)}-\alpha_{\pi(1)} \leq|a|-a_{\pi(n)}+1 .
$$

Notice that the first inequality is strict if $\pi$ is not the identity permutation, while the second inequality is strict if $\pi(n) \neq 1$. Suppose that $\pi(n)=1$. Then there is exactly one $i$ such that $\pi(i+1)<\pi(i)$. This implies that $\pi=(2,3, \ldots, n, 1)$ and $\alpha_{r}=a_{2}+\cdots+a_{r-1}$, which is not possible in view of the choice of $B_{r}$. Thus $\pi(n) \neq 1$, implying $\pi=$ id and $\alpha_{i}=a_{1}+\cdots+a_{i-1}$ for every $i$. Substituting these values into

$$
\frac{F\left(c_{1}, c_{2}, \ldots, c_{n}\right)}{\phi_{1}^{\prime}\left(c_{1}\right) \phi_{2}^{\prime}\left(c_{2}\right) \cdots \phi_{n}^{\prime}\left(c_{n}\right)}
$$

one recovers the case $s=1$ of Theorem 5.2 without any difficulty.

As a final remark we mention that Zhou 38 successfully applied Theorem 5.1 to prove another conjecture of Kadell [20, Conjecture 2] related to the Dyson product. The referee also asked if our results could be used to obtain Zhou's theorem. At present we do not know how to do this. However, the polynomial method can be used to prove a special case of Kadell's [20, Conjecture 3]; see [23].

\section{ACKNOWLEDGEMENT}

The authors thank David Bressoud for helpful correspondence and the anonymous referee for raising some interesting questions, leading to the material presented in Section 5 .

\section{REFERENCES}

[1] N. Alon, Combinatorial Nullstellensatz, Combin. Probab. Comput. 8 (1999), no. 1-2, 7 29, DOI 10.1017/S0963548398003411. Recent trends in combinatorics (Mátraháza, 1995). MR.1684621 (2000b:05001)

[2] G. E. Andrews, Problems and prospects for basic hypergeometric functions, Theory and application of special functions (Proc. Advanced Sem., Math. Res. Center, Univ. Wisconsin, Madison, Wis., 1975), Academic Press, New York, 1975, pp. 191-224. Math. Res. Center, Univ. Wisconsin, Publ. No. 35. MR0399528 (53 \#3372)

[3] G. E. Andrews, The theory of partitions, Addison-Wesley Publishing Co., Reading, Mass.London-Amsterdam, 1976. Encyclopedia of Mathematics and its Applications, Vol. 2. MR.0557013 (58 \#27738)

[4] C. Berge, Principles of combinatorics, Translated from the French. Mathematics in Science and Engineering, Vol. 72, Academic Press, New York, 1971. MR0270922 (42 \#5805)

[5] N. Bourbaki, Lie groups and Lie algebras. Chapters 4-6, Elements of Mathematics (Berlin), Springer-Verlag, Berlin, 2002. Translated from the 1968 French original by Andrew Pressley. MR.1890629(2003a:17001)

[6] D. M. Bressoud and I. P. Goulden, Constant term identities extending the $q$-Dyson theorem, Trans. Amer. Math. Soc. 291 (1985), no. 1, 203-228, DOI 10.2307/1999904. MR797055 (86k:05011)

[7] R. A. Brualdi and H. J. Ryser, Combinatorial matrix theory, Encyclopedia of Mathematics and its Applications, vol. 39, Cambridge University Press, Cambridge, 1991. MR1130611 (93a:05087)

[8] I. Cherednik, The Macdonald constant-term conjecture, Internat. Math. Res. Notices 6 (1993), 165-177, DOI 10.1155/S1073792893000170. MR1224114 (94i:17016)

[9] I. Cherednik, Double affine Hecke algebras and Macdonald's conjectures, Ann. of Math. (2) 141 (1995), no. 1, 191-216, DOI 10.2307/2118632. MR1314036 (96m:33010)

[10] I. Cherednik, Double affine Hecke algebras, London Mathematical Society Lecture Note Series, vol. 319, Cambridge University Press, Cambridge, 2005. MR2133033 (2007e:32012) 
[11] C. Chevalley, Sur certains groupes simples (French), Tôhoku Math. J. (2) 7 (1955), 14-66. $\operatorname{MR} 0073602(17,457 \mathrm{c})$

[12] M. Demazure, Une nouvelle formule des caractères, Bull. Sci. Math. (2) 98 (1974), no. 3, 163-172. MR0430001 (55 \#3009)

[13] P. J. Forrester and S. O. Warnaar, The importance of the Selberg integral, Bull. Amer. Math. Soc. (N.S.) 45 (2008), no. 4, 489-534, DOI 10.1090/S0273-0979-08-01221-4. MR2434345 $(2009 \mathrm{~m}: 33001)$

[14] A. M. Fu and A. Lascoux, Non-symmetric Cauchy kernels for the classical groups, J. Combin. Theory Ser. A 116 (2009), no. 4, 903-917, DOI 10.1016/j.jcta.2008.10.007. MR2513641 (2010f:05190)

[15] G. Gasper and M. Rahman, Basic hypergeometric series, 2nd ed., Encyclopedia of Mathematics and its Applications, vol. 96, Cambridge University Press, Cambridge, 2004. With a foreword by Richard Askey. MR2128719 (2006d:33028)

[16] I. M. Gessel and G. Xin, A short proof of the Zeilberger-Bressoud q-Dyson theorem, Proc. Amer. Math. Soc. 134 (2006), no. 8, 2179-2187 (electronic), DOI 10.1090/S0002-9939-0608224-4. MR2213689 (2007e:05012)

[17] J. E. Humphreys, Introduction to Lie algebras and representation theory, Springer-Verlag, New York, 1972. Graduate Texts in Mathematics, Vol. 9. MR0323842 (48 \#2197)

[18] J. E. Humphreys, Reflection groups and Coxeter groups, Cambridge Studies in Advanced Mathematics, vol. 29, Cambridge University Press, Cambridge, 1990. MR.1066460 (92h:20002)

[19] B. Ion, Nonsymmetric Macdonald polynomials and Demazure characters, Duke Math. J. 116 (2003), no. 2, 299-318, DOI 10.1215/S0012-7094-03-11624-5. MR.1953294 (2004d:33019)

[20] K. W. J. Kadell, Aomoto's machine and the Dyson constant term identity, Methods Appl. Anal. 5 (1998), no. 4, 335-350. MR.1669871 (2000m:33018)

[21] K. W. J. Kadell, A Dyson constant term orthogonality relation, J. Combin. Theory Ser. A 89 (2000), no. 2, 291-297, DOI 10.1006/jcta.1999.2928. MR.1741011(2001h:05021)

[22] R. N. Karasev and F. V. Petrov, Partitions of nonzero elements of a finite field into pairs, Israel J. Math. 192 (2012), no. 1, 143-156, DOI 10.1007/s11856-012-0020-5. MR3004078

[23] G. Károlyi, Z. L. Nagy, F. V. Petrov, and V. Volkov, A new approach to constant term identities and Selberg-type integrals, Adv. Math. 277 (2015), 252-282, DOI 10.1016/j.aim.2014.09.028. MR.3336087

[24] G. Károlyi and Z. L. Nagy, A simple proof of the Zeilberger-Bressoud q-Dyson theorem, Proc. Amer. Math. Soc. 142 (2014), no. 9, 3007-3011, DOI 10.1090/S0002-9939-2014-120417. MR3223356

[25] A. Lascoux, Symmetric functions and combinatorial operators on polynomials, CBMS Regional Conference Series in Mathematics, vol. 99, Published for the Conference Board of the Mathematical Sciences, Washington, DC, 2003. MR2017492 (2005b:05217)

[26] A. Lascoux and M.-P. Schützenberger, Keys \& standard bases, Invariant theory and tableaux (Minneapolis, MN, 1988), IMA Vol. Math. Appl., vol. 19, Springer, New York, 1990, pp. 125144. MR1035493 (91c:05198)

[27] M. Lasoń, A generalization of combinatorial Nullstellensatz, Electron. J. Combin. 17 (2010), no. 1, Note 32, 6. MR2729390(2012c:05352)

[28] L. Lv, G. Xin, and Y. Zhou, A family of q-Dyson style constant term identities, J. Combin. Theory Ser. A 116 (2009), no. 1, 12-29, DOI 10.1016/j.jcta.2008.04.002. MR2469245 (2009k:05032)

[29] I. G. Macdonald, The Poincaré series of a Coxeter group, Math. Ann. 199 (1972), 161-174. MR.0322069(48 \#433)

[30] I. G. Macdonald, Some conjectures for root systems, SIAM J. Math. Anal. 13 (1982), no. 6, 988-1007, DOI 10.1137/0513070. MR674768(84h:17006a)

[31] I. G. Macdonald, Symmetric functions and Hall polynomials, 2nd ed., Oxford Mathematical Monographs, The Clarendon Press Oxford University Press, New York, 1995. With contributions by A. Zelevinsky; Oxford Science Publications. MR 1354144 (96h:05207)

[32] A. V. Sills, Disturbing the Dyson conjecture, in a generally GOOD way, J. Combin. Theory Ser. A 113 (2006), no. 7, 1368-1380, DOI 10.1016/j.jcta.2005.12.005. MR2259066 (2007g:05016)

[33] L. Solomon, The orders of the finite Chevalley groups, J. Algebra 3 (1966), 376-393. MR0199275 (33 \#7424) 
[34] R. P. Stanley, Enumerative combinatorics. Vol. 1, Cambridge Studies in Advanced Mathematics, vol. 49, Cambridge University Press, Cambridge, 1997. With a foreword by Gian-Carlo Rota; corrected reprint of the 1986 original. MR 1442260 (98a:05001)

[35] J. R. Stembridge, First layer formulas for characters of $\operatorname{SL}(n, \mathbf{C})$, Trans. Amer. Math. Soc. 299 (1987), no. 1, 319-350, DOI 10.2307/2000497. MR869415 (88g:20088)

[36] J. R. Stembridge, On the Poincaré series and cardinalities of finite reflection groups, Proc. Amer. Math. Soc. 126 (1998), no. 11, 3177-3181, DOI 10.1090/S0002-9939-98-04473-6. MR 1459151(99a:20052)

[37] D. Zeilberger and D. M. Bressoud, A proof of Andrews' q-Dyson conjecture, Discrete Math. 54 (1985), no. 2, 201-224, DOI 10.1016/0012-365X(85)90081-0. MR791661 (87f:05015)

[38] Y. Zhou, On Kadell's two conjectures for the q-Dyson product, Electron. J. Combin. 18 (2011), no. 2, Paper 2, 15. MR2770132(2012b:05040)

School of Mathematics and Physics, The University of Queensland, Brisbane, QLD 4072, Australia - And - Institute of Mathematics, EÖtvös University, Budapest, HunGARY

CnRs, Institut Gaspard Monge, Université Paris-Est, Marne-la-Vallée, France

School of Mathematics and Physics, The University of Queensland, Brisbane, QLD 4072, Australia 